\title{
Waterlogging Risk Assessment of the Beijing- Tianjin-Hebei Urban Agglomeration in the Past 60 Years
}

\section{Yujie Wang}

Nanjing University of Information Science and Technology

JIANQING ZHAI ( $\square$ zhaijq@cma.gov.cn )

National Climate Center, CMA https://orcid.org/0000-0001-7793-3966

Lianchun Song

National Climate Center, CMA

\section{Research Article}

Keywords: Hazard, Exposure, Vulnerability, Waterlogging risk, Beijing-Tianjin-Hebei

Posted Date: February 10th, 2021

DOI: https://doi.org/10.21203/rs.3.rs-162526/v1

License: (c) (i) This work is licensed under a Creative Commons Attribution 4.0 International License.

Read Full License 
1 Waterlogging Risk Assessment of the Beijing-TianjinHebei Urban Agglomeration in the Past 60 Years

3

4

\author{
Yujie Wang ${ }^{1,2}$, Jianqing Zhai ${ }^{\square 3}$, Lianchun Song ${ }^{3}$
}

1 Key Laboratory of Meteorological Disaster, Ministry of Education/International Joint Research

Laboratory on Climate and Environment Change/Collaborative Innovation Center on Forecast and Evaluation of Meteorological Disasters, Nanjing University of Information Science and Technology, Nanjing 210044, China

2 School of Atmospheric Sciences, Nanjing University of Information Science and Technology, Nanjing 210044, China

3 National Climate Center, CMA, Beijing 100081, China

$\square$ Corresponding author: Jianqing Zhai

E-mail: Zhaijq@cma.gov.cn 
In the context of global climate change and rapid urbanization, the risk of urban waterlogging is one of the main climate risks faced by the Beijing-Tianjin-Hebei (BTH) urban agglomeration. In this study, we obtain the urban waterlogging risk index of the BTH urban agglomeration and assess waterlogging risks in the built-up area of the BTH for two time periods (1961-1990 and 1991-2019). We analyze the economic and social data as well as the climate data from 149 meteorological observation stations in Beijing,

Tianjin, and Hebei provinces and consider the hazard, exposure, and vulnerability factors. The results showed that for the two time periods considered, the areas with the lowest (Level-1) waterlogging risk have decreased by nearly $50 \%$, and the secondlowest (Level-2) ones have increased by nearly 55\%. Although the areas at Level-3 and above have decreased by $17 \%$, the variations in each city were quite different. Among them, the areas at Level-3 have increased by 52\% and 51\% in Beijing and Handan, respectively. During the years 1991-2019, the areas at Level-3 risk and above were mainly found in Beijing, Tianjin, Shijiazhuang, Qinhuangdao, Handan, and Xingtai. Among them, Beijing had the highest waterlogging risk index. In particular, the areas with Level-3 risk and above have increased by $76 \%$ in the past 30 years. The areas with the highest risk level (Level-4) of waterlogging in Beijing were mostly found in the downtown areas (Haidian, Chaoyang, Dongcheng, and Xicheng districts). This study provides a scientific background for urban waterlogging risk management and implementation of the national strategy for the development of the BTH region.

Keywords: Hazard, Exposure, Vulnerability, Waterlogging risk, Beijing-Tianjin-Hebei 


\section{Introduction}

China is undergoing rapid urbanization. By the end of 2016, there were 88 large cities in the country with over 1 million inhabitants. The cities with dense population, concentrated assets, high exposure, and vulnerability are at high risk for climate change (Zhai et al., 2018). In the context of climate change and urbanization, the changes and impacts of extreme precipitation in cities have attracted more and more attention ( $\mathrm{Li}$ et al., 2015; Zhou et al., 2017; Bai et al., 2018; Gu et al., 2019). Due to the hardening of the underlying urban surface and poor drainage facilities, heavy rainfalls will increase the risk of urban waterlogging (Kaźmierczak et al., 2011; Wang et al., 2014; Yu et al., 2018a).

The Beijing-Tianjin-Hebei (BTH) region is one of the three fastest-growing megacity clusters in China, with an area of $216,000 \mathrm{~km}^{2}$ and a population of 110 million, including 13 cities (Beijing, Tianjin, Shijiazhuang, Baoding, Tangshan, Langfang, Handan, Qinhuangdao, Zhangjiakou, Chengde, Cangzhou, Xingtai, and Hengshui). The precipitation in the BTH cluster is uneven during the year, varies from year to year, and features heavy rainstorm intensity (Zheng et al., 2018). Recent decades have witnessed the increase of the average temperature, the decrease of the annual precipitation, and the number of precipitation days (Zhai et al., 2005; Li et al., 2011; Wang et al., 2020). More specifically, the frequency and intensity of extreme summer precipitation have increased (Zhang et al., 2010; Liang et al., 2018). In particular, the hour-scale extreme heavy rainfall occurs more often, leading to prominent urban waterlogging problems (Yin et al., 2011; Yang et al., 2014; Song et al., 2014; Liang et al., 2017). For example, on July 21, 2012, the 18-hour maximum rainfall in Beijing reached $460 \mathrm{~mm}$, which caused serious urban flooding. The disaster covered $16,000 \mathrm{~km}^{2}$, affected 1.9 million populations, left 79 people dead, and paralyzed the traffic (NCC, 2013). For the continuous global warming, the intensity of extreme precipitation events in the BTH urban agglomeration will further increase (Yu et al., 2018b; Shi et al., 2019) and will cause serious risks of urban waterlogging.

Recently, urban waterlogging risks have been extensively studied (Saeed et al., 2010; Li et al., 2015; Hu, 2016; Wang et al., 2017; Wang et al., 2018). Various problems have 
been considered ranging from urban rainwater runoff modeling to the construction of urban waterlogging simulation system $(\mathrm{Li}, 2002)$ and the two-dimensional hydrodynamic evaluation model (Xie et al., 2018). However, most of the researchers focused on assessing the waterlogging risks in a single city. The same problem for multiple cities at the regional scale was commonly addressed using the data from the weather station located in a given urban area. This approach could not reflect the spatial difference in the urban waterlogging risk at the regional scale (Zheng et al., 2018; Sun et al., 2020). Therefore, there is currently a lack of a regional-scale urban waterlogging risk assessment system featuring high spatial resolution and even fewer studies on waterlogging risks and changes in the BTH urban agglomeration.

This paper addresses the problem mentioned above for the urban built-up BTH area. More specifically, we consider hourly precipitation, daily precipitation, and 5-day continuous precipitation as the hazard factors of a rainstorm and waterlogging, population density and GDP per unit area as exposure indicators, and adaptability to heavy rainfall and waterlogging such as drainage network density, vegetation coverage and river network density as vulnerability indicators. The waterlogging risks in the built-up area of the BTH urban agglomeration during the two periods of 1961 to 1990 and 1991 to 2019 were assessed, aiming to reveal the characteristics of waterlogging risk changes in the past 60 years. Thus, we aimed to provide scientific background for the implementation of the national strategy for the coordinated development of the BTH region.

\section{Data and Methods}

\subsection{Data}

The meteorological data used in this paper came from 149 meteorological observation stations in the BTH region provided by the National Meteorological Information Center of the China Meteorological Administration. The hourly and daily precipitation data from 1961 to 2019 (Figure 1) have undergone quality control and uniformity inspection (Ren et al., 2012).

The population and GDP grid data at $1 \mathrm{~km}$ resolution of the BTH region in 2015 came 
from the Data Center for Resources and Environmental Sciences of Chinese Academy of Sciences (http://www.resdc.cn/Default.aspx). We developed the population and GDP grid data at $1 \mathrm{~km}$ resolution of the BTH region in 1990(Fu et al., 2014) based on the population and GDP statistics of the BTH region from China City Statistical Yearbook 1991, and referring to the data processing method of the Data Center for Resources and Environmental Sciences of Chinese Academy of Sciences.

The spatial distribution, land usage, and river network density data of 2015 in 13 cities of the BTH came from Tsinghua University with a spatial resolution of 30 meters (http://data.ess.tsinghua.edu.cn), and drainage data including pipeline length and vegetation coverage area was obtained from the China City Statistical Yearbook 2016.

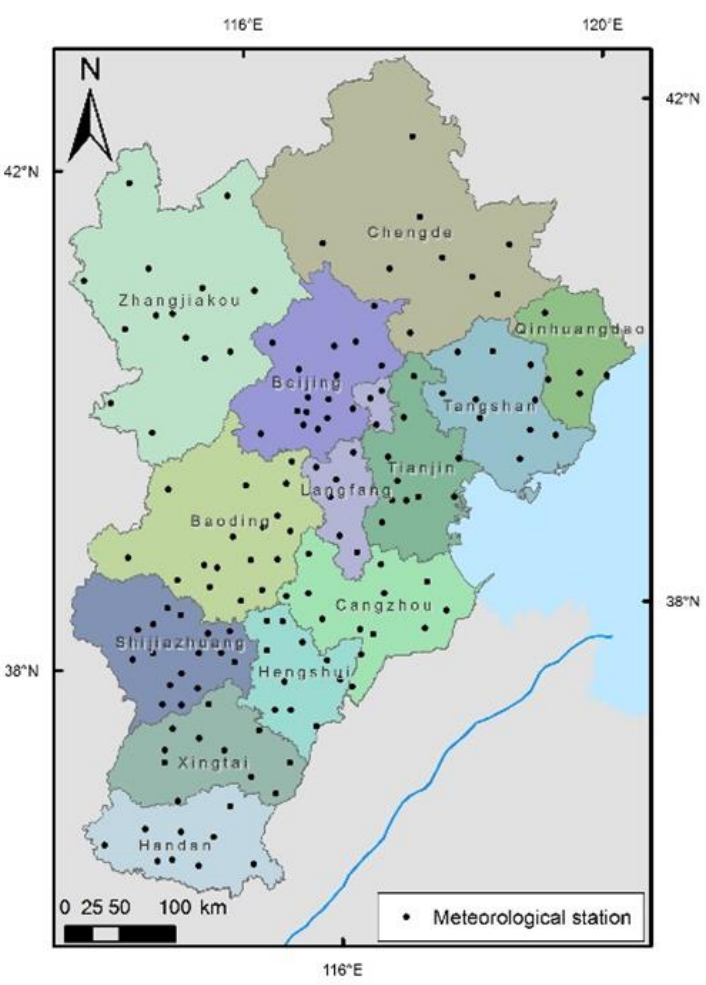

Figure 1 Administrative divisions and meteorological stations in the BTH.

\subsection{Methods}

\subsubsection{Normalization method}

To normalize all the factors considered in this study, we used the following expression:

$$
\mathrm{I}=5+\left(\mathrm{X}_{\mathrm{i}}-\mathrm{X}_{\mathrm{a}}\right) / \sigma
$$

Here, $\mathrm{I}$ is the normalized value, $\mathrm{X}_{\mathrm{i}}$ is the value of the $i$-th factor, $\mathrm{X}_{\mathrm{a}}$ is the mean value 
of this factor, and $\sigma$ stands for its standard deviation.

\subsubsection{Waterlogging risk index}

We followed IPCC AR5 (IPCC, 2014) and used a three-determinants risk model. We consider hazard, exposure, and vulnerability as model parameters and accounted for the recently suggested method of Sun et al. (2020). The resulting equation for waterlogging risk assessment reads as:

$$
\mathrm{R}=\mathrm{H} \times \mathrm{E} \times(10-\mathrm{A})
$$

here, $\mathrm{H}$ is the hazard index, $\mathrm{E}$ is the exposure index, and $\mathrm{A}$ is the adaptability index.

The waterlogging risk was divided into four grades according to the standard deviation $(\sigma)$ ranging from 1 to 4 , with values from low to very high, respectively. The standards were set as follows:

low risk (Level 1): $\mathrm{R} \leqslant \mathrm{R}_{\mathrm{a}}-0.5 \sigma$;

moderate risk (Level 2): $\mathrm{R}_{\mathrm{a}}-0.5 \sigma<\mathrm{R} \leqslant \mathrm{R}_{\mathrm{a}}+0.5 \sigma$;

high risk (Level 3): $\mathrm{R}_{\mathrm{a}}+0.5 \sigma<\mathrm{R} \leqslant \mathrm{R}_{\mathrm{a}}+\sigma$;

very high risk (Level 4): $\mathrm{R}>\mathrm{R}_{\mathrm{a}}+\sigma$.

Here, $\mathrm{R}$ is the waterlogging risk level, and $\mathrm{R}_{\mathrm{a}}$ is the average value of $\mathrm{R}$.

\subsubsection{Hazard index}

We adopted a maximum of 1 hour, 1 day, and 5 continuous days precipitation as the waterlogging hazard factors. Since most of the precipitation data from the meteorological stations in the BTH region covered the last 60 years and considering the uncertainty of the return period calculation, we used the common practice adopted for the extreme climate studies. More specifically, we calculated the hazard index for every 20 years (Kharin et al., 2013, Sillmann et al., 2013). The hazard assessment was divided into two time periods from 1961 to 1990 and from 1991 to 2019, to analyze the variation characteristics under the background of climate change. The maximum precipitation in 1 hour, 1 day, and 5 days for these two time periods once in every 20 years were calculated using the generalized extreme value distribution (GEV) method (Chen et al., 2010). Then, the normalized hazard index was given by:

$$
\mathrm{D}=0.2 \times H_{1 h}+0.6 \times H_{1 d}+0.2 \times H_{5 d},
$$

where $\mathrm{D}$ is the hazard index, $H_{1 h}, H_{1 d}$, and $H_{5 d}$ stand for the maximum precipitation 
in 1 hour, 1 day, and 5 days once in every 20 years. The weights of different hazard factors were scored by subject matter experts. The hazard index referred to the risk level of waterlogging and was divided into four levels: low, medium, high, and very high, ranging from 1 to 4 .

\subsubsection{Exposure index}

The spatial data from the BTH region for the year 2015 was processed, and the resolution was scaled up to $1 \mathrm{~km}$ using the Arcmap resampling method. The grid data population and GDP were obtained, combining with the population and economic data for the years 1990 and 2015. The normalized exposure indices in 1990 and 2015 were determined from (Sun et al., 2020):

$$
\mathrm{E}=0.5 \times \mathrm{P}+0.5 \times \mathrm{G}
$$

Here, $\mathrm{P}$ and $\mathrm{G}$ are the grid population and grid economic factors, respectively.

The exposure index was divided into four levels: low, medium, high, and very high according to the risk level of waterlogging, ranging from 1 to 4 .

\subsubsection{Adaptability index}

The vulnerability was characterized by the index of the city's adaptability to rainstorm and waterlogging. The adaptability to rainstorm and waterlogging comprehensively considered the density of urban drainage pipe network, vegetation coverage, and river network density.

The drainage network density and vegetation coverage rate of the built-up area were spatialized based on the urban drainage pipeline length and vegetation coverage in the Statistical Yearbook 2015, combined with the grid distribution data. Based on the land usage data at 30-meter resolution in 2015 , the river network density information was resampled to a $1 \mathrm{~km}$ grid.

Expert scoring showed that all these three factors played a positive role in urban waterlogging and had the same contribution rate. The density of drainage pipe network, vegetation coverage, and river network density were all normalized and divided into four levels of the low, medium, high, and very high, ranging from 1 to 4 . The equal weight sum method was adopted to obtain the urban waterlogging adaptability index given by: 


$$
\mathrm{A}=(\mathrm{D}+\mathrm{V}+\mathrm{R}) / 3 \text {, }
$$

175

where $\mathrm{D}, \mathrm{V}$, and $\mathrm{R}$ stand for the density of drainage pipe network, the vegetation coverage rate, and the density of the river network.

The adaptability index was divided into four levels, low, medium, high, and very high, ranging from 1 to 4.

\section{Results}

\subsection{Hazard}

\subsubsection{One-hour precipitation distribution}

In the past 60 years, the distribution of one-hour precipitation, $H_{1 h}$, in the $\mathrm{BTH}$ decreased from southeast to northwest. For most areas in the south of the Taihang Mountains, it exceeded $60 \mathrm{~mm}$, while the northern areas generally had less than $50 \mathrm{~mm}$. The comparison of time periods 1961-1990 and 1991-2019 revealed that the percentage of $H_{1 h}$ over $60 \mathrm{~mm}$ increased significantly in Beijing, northern Tianjin, northern Shijiazhuang, and southern Qinhuangdao, while decreasing in Baoding (Figure 2). More specifically, the maximum $H_{1 h}$ in Beijing has increased from 72.8 $\mathrm{mm}$ to $96.8 \mathrm{~mm}(33.0 \%)$ and the average $H_{1 \mathrm{~h}}$ increased from $54.5 \mathrm{~mm}$ to $61.9 \mathrm{~mm}$ $(13.6 \%)$. At the same time, the maximum $H_{1 h}$ in Baoding decreased from $93.1 \mathrm{~mm}$ to $76.0 \mathrm{~mm}$. This change in short-time heavy precipitation was likely caused by the urban heat islands that enhanced the vertical mixing of urban water vapor. In particular, the strong convective system was split in the upwind direction of the city and merged in the downwind one. The latter increased heavy precipitation in the urban and downwind directions (Zhang et al., 2009; Zhang et al.,2014; Yang et al., 2017). 
197
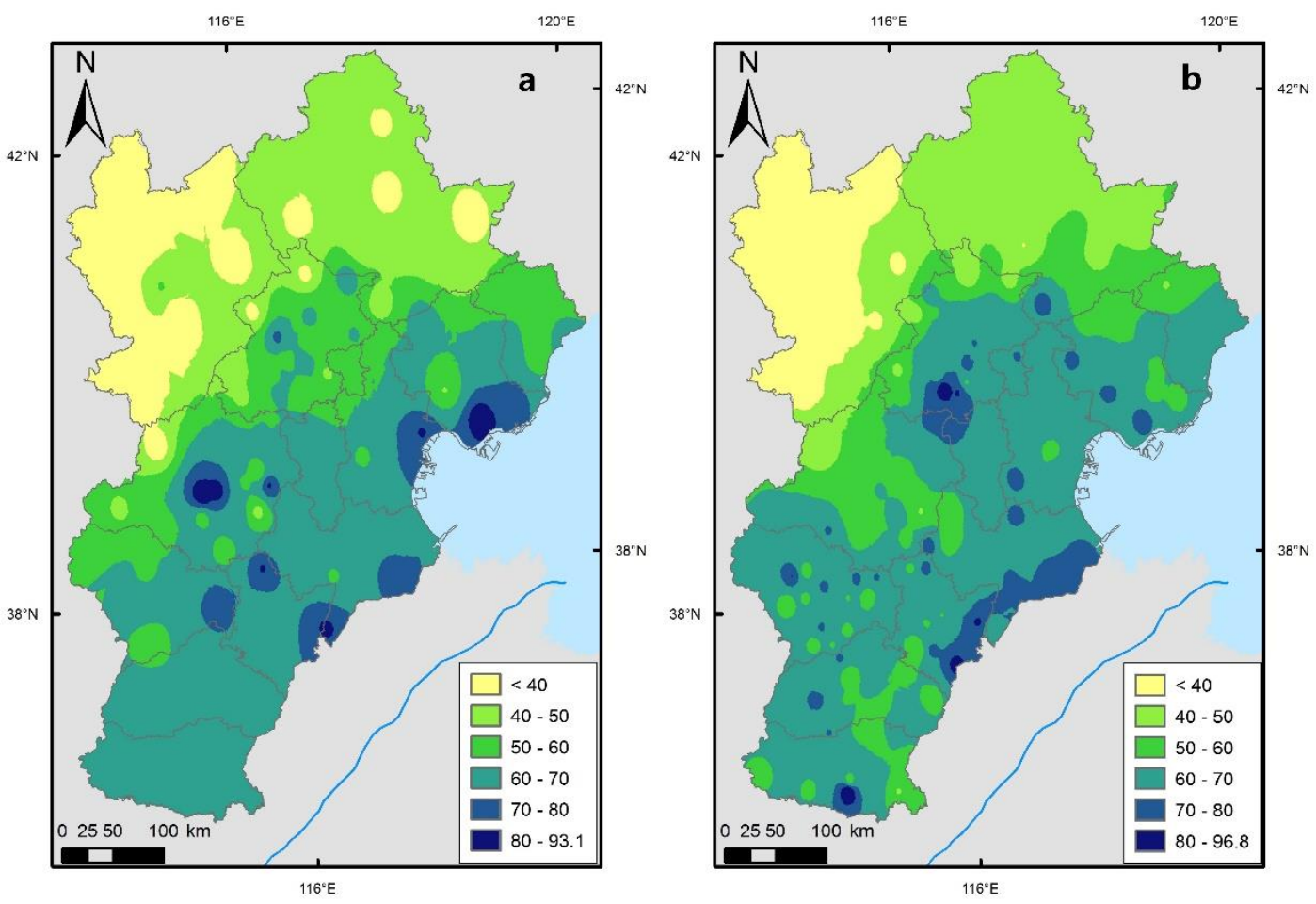

Figure 2 One-hour precipitation distribution in the BTH for (a) 1961-1990 and (b) 1991-2019 time periods.

\subsubsection{One-day precipitation distribution}

Unlike one-hour distribution, the daily one, $H_{1 d}$, exceeding $150 \mathrm{~mm}$ decreased dramatically from 1961-1990 to 1991-2019 time period. Moreover, regional distribution has also changed. Previously (1961-1990), it has been mainly distributed in Tianjin, Tangshan, Qinhuangdao, Cangzhou, Hengshui, eastern Langfang, central Baoding, southwestern Chengde, eastern Handan, and most parts of Xingtai. However, in the years 1991-2019, the distribution has changed for southern Beijing, most part of Tianjin, Langfang, Cangzhou, Qinhuangdao, western Shijiazhuang, northeastern Baoding, western Xingtai, and western Handan (Figure 3). In the past 30 years, the maximum $H_{1 d}$ in the BTH decreased from $270.6 \mathrm{~mm}$ to $229.0 \mathrm{~mm}$ (18.2\%), but the one in Beijing increased from $198.8 \mathrm{~mm}$ to $229.9 \mathrm{~mm}$ (15.6\%). Moreover, the maximum $H_{1 d}$ of Tangshan has decreased from $270.6 \mathrm{~mm}$ to $179.6 \mathrm{~mm}(50.7 \%)$ along with that of Shijiazhuang, Baoding, and Zhangjiakou. At the same time, one-day maximums for Qinhuangdao, Tianjin, Chengde, Cangzhou, and Hengshui have dropped dramatically. 
214
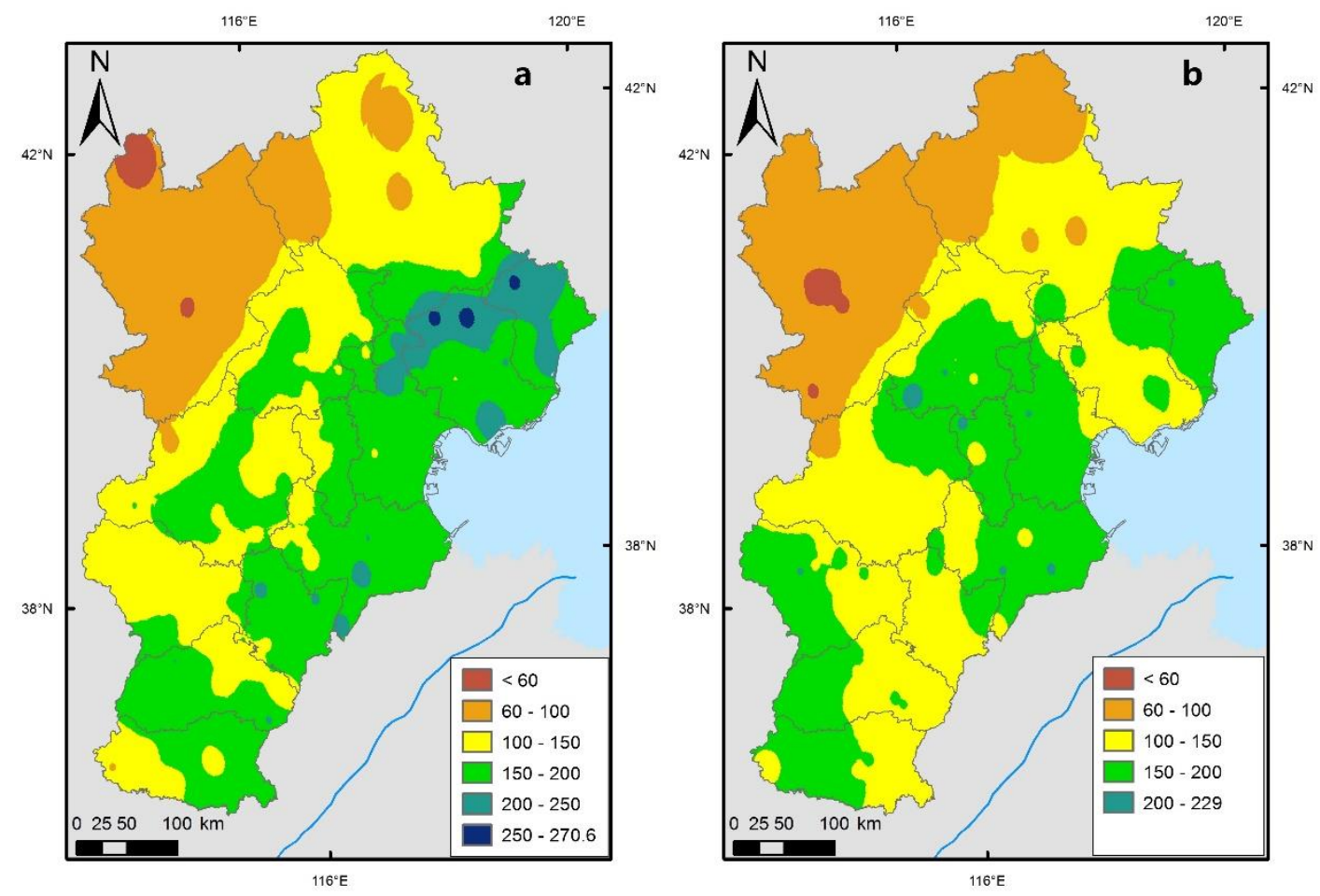

Figure 3 One-day precipitation distribution in the BTH for (a) 1961-1990 and (b) 1991-2019 time periods.

\subsubsection{Five-day precipitation distribution}

Similar to one-day distribution, the area with precipitation above $250 \mathrm{~mm}$ for fiveday one, $H_{5 d}$, in the BTH region has decreased significantly from 1961-1990 to 19912019 (Figure 4). Moreover, and the maximum $H_{5 d}$ of the area has also decreased from $540.1 \mathrm{~mm}$ to $354.7 \mathrm{~mm}$ (52.3\%). In particular, except for Shijiazhuang, where maximum $H_{5 d}$ has increased from $315.2 \mathrm{~mm}$ to $354.7 \mathrm{~mm}(15 \%)$, it has decreased for all other cities, with the largest decrease occurred in Tianjin (from $521.6 \mathrm{~mm}$ to $251.0 \mathrm{~mm}$ or by $107.8 \%)$. The decrease of continuous precipitation in the BTH may be related to the weakening of the East Asian summer monsoon, which reduced the water vapor flux into Northern China from the southern border, and the southward shift of the subtropical high (Hao et al., 2011). 
230
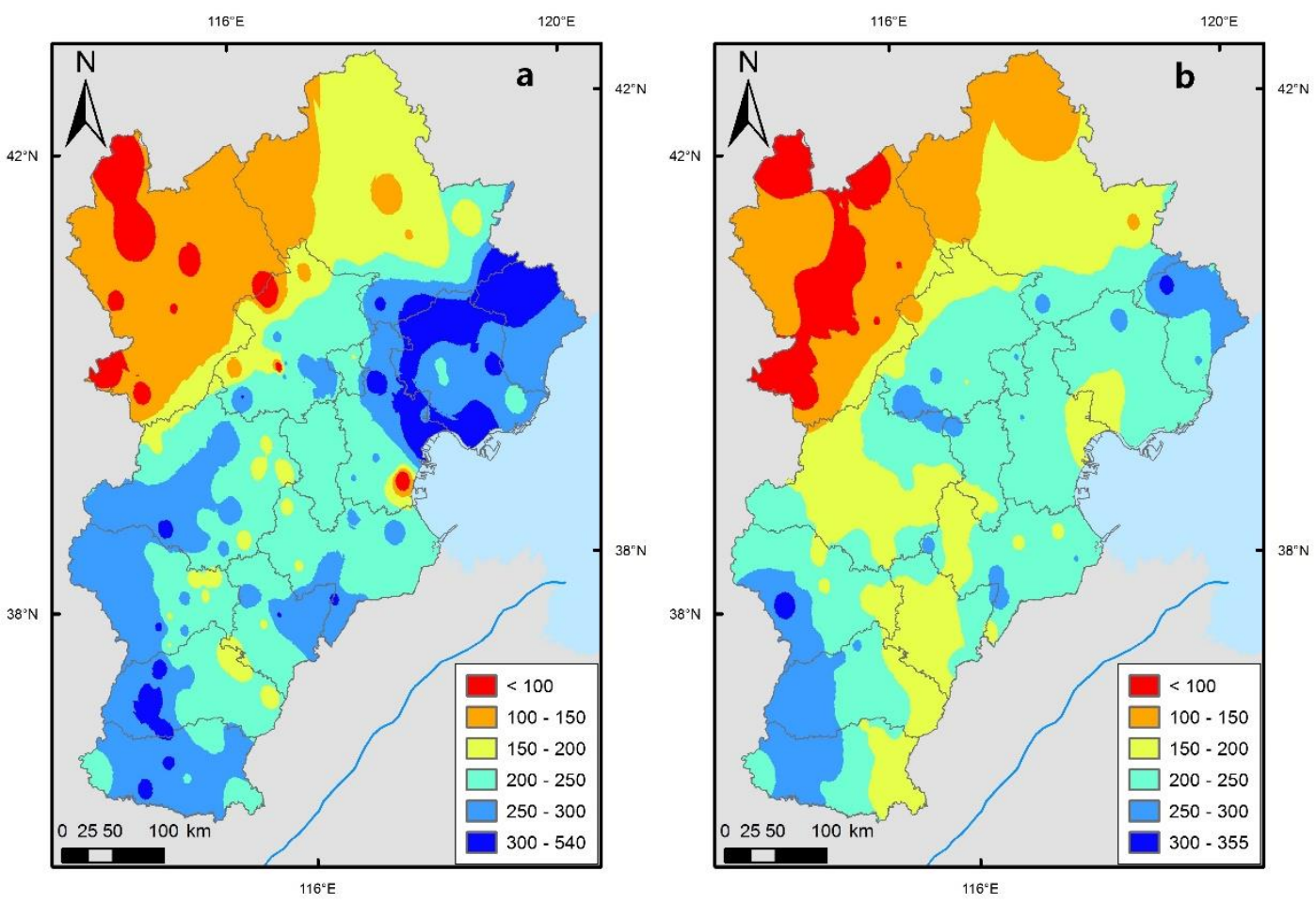

Figure 4 Five-day precipitation distribution in the BTH for (a) 1961-1990 and (b) 1991-2019 time periods.

\subsubsection{Hazard index}

The risk index can be calculated from (3) after normalizing $H_{1 h}, H_{1 d}$ and $H_{5 d}$. The corresponding results are shown in Figure 5 and demonstrate that the risks for Zhangjiakou and northern Chengde were at risk Level-1 during both time periods. The areas with Level-3 risk and above during the 1961-1990 time period included Qinhuangdao, Tangshan, Tianjin, Cangzhou, Hengshui, western Xingtai, eastern Handan, and central Baoding. The areas with Level-4 risks were located in Qinhuangdao, Tangshan, and other places. The situation has changed for the time period of 1991-2019. In particular, the areas with Level-3 risk and above were mainly in Beijing, Tianjin, western Shijiazhuang, Qinhuangdao, Langfang, Cangzhou, the eastern Tangshan, western Handan, and western Xingtai. Among them, the areas with Level-4 risk included southern Beijing, western Tianjin, western Shijiazhuang, southern Cangzhou, and western Xingtai. Compared to the time period from 1961 to 1990, the risk indices of Beijing, Shijiazhuang, and Langfang have increased significantly in the 
past 30 years (by $41 \%, 42 \%$, and $35 \%$, respectively). At the same time, the risk indices of Tangshan and Hengshui have decreased significantly (by $29 \%$ and $21 \%$, respectively, Table 1).
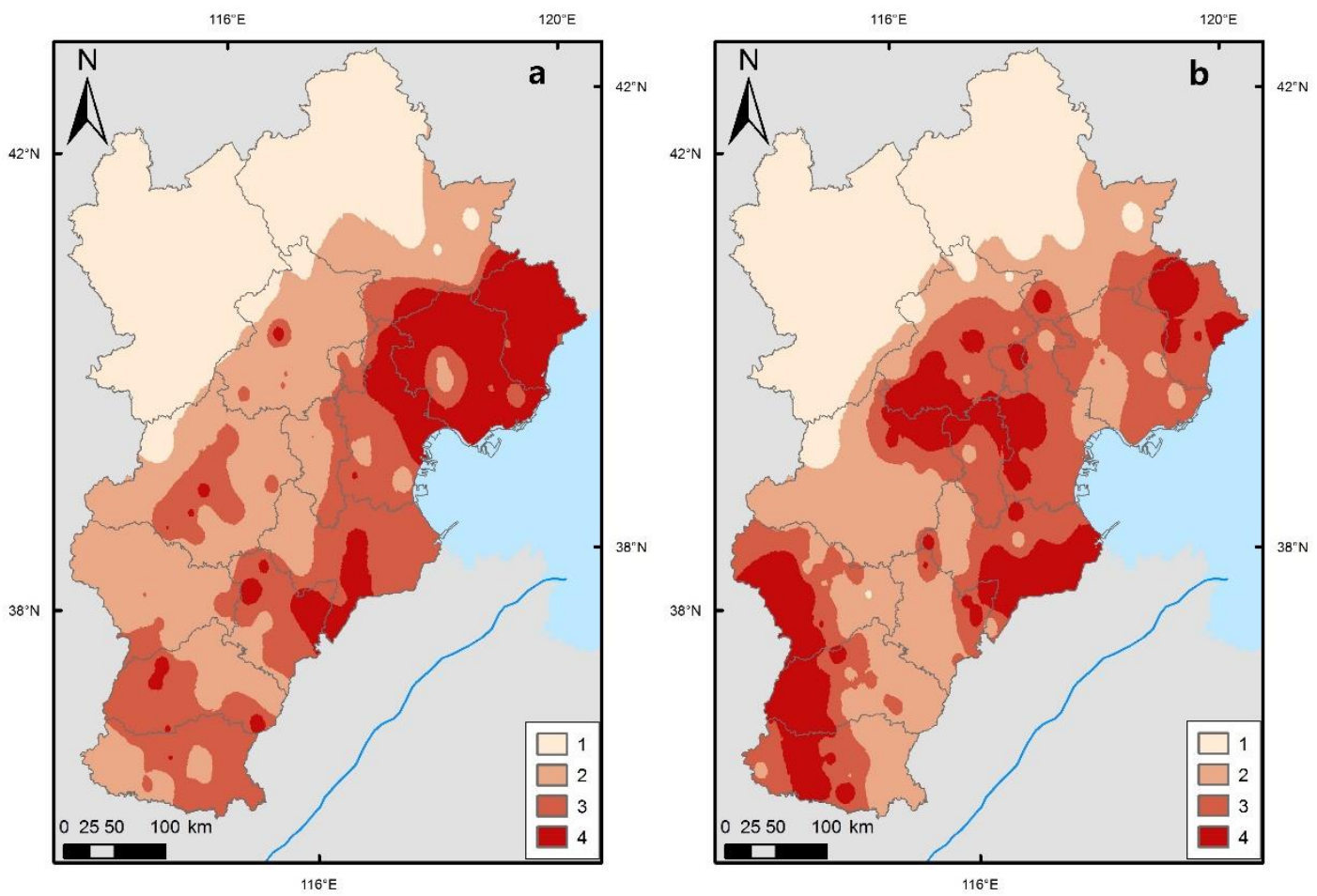

Figure 5 Hazard intensity for (a) 1961-1990 and (b) 1991-2019 time periods. It was measured once in every 20 years. Numbers is legend correspond to hazard intensity levels.

\subsection{Exposure index}

To reveal the dynamics of changes in exposure index for urban built-up areas of BTH, we selected the years 1990 and 2015 as the representatives of the two periods (19611990 and 1991-2019) and analyzed it. As shown in Figure 6, in 1990 and 2015, the areas of Beijing, Tianjin, and Qinhuangdao had Level-4 exposure and demonstrated more than $11.2 \%$ increase. Among them, the area of Beijing and Tianjin at Level-3 exposure risk and above and increased from $25.2 \%$ and $22.5 \%$ in 1990 to $47.3 \%$ and $58.2 \%$ in 2015, respectively. At the same time, the exposure for Tangshan, Zhangjiakou, and Chengde decreased from $17.8 \%, 12.7 \%$, and $18.7 \%$ in 1990 to $3.5 \%, 2.7 \%$, and $0.4 \%$ in 2015 , respectively. The exposure of other cities was relatively low. It can be seen that the development of the BTH urban agglomeration came in line with the concentration of population, capital, and materials in the megacities of Beijing and 
Tianjin. Among the changes in the average exposure index of each city, Beijing had the largest increase of $32 \%$.
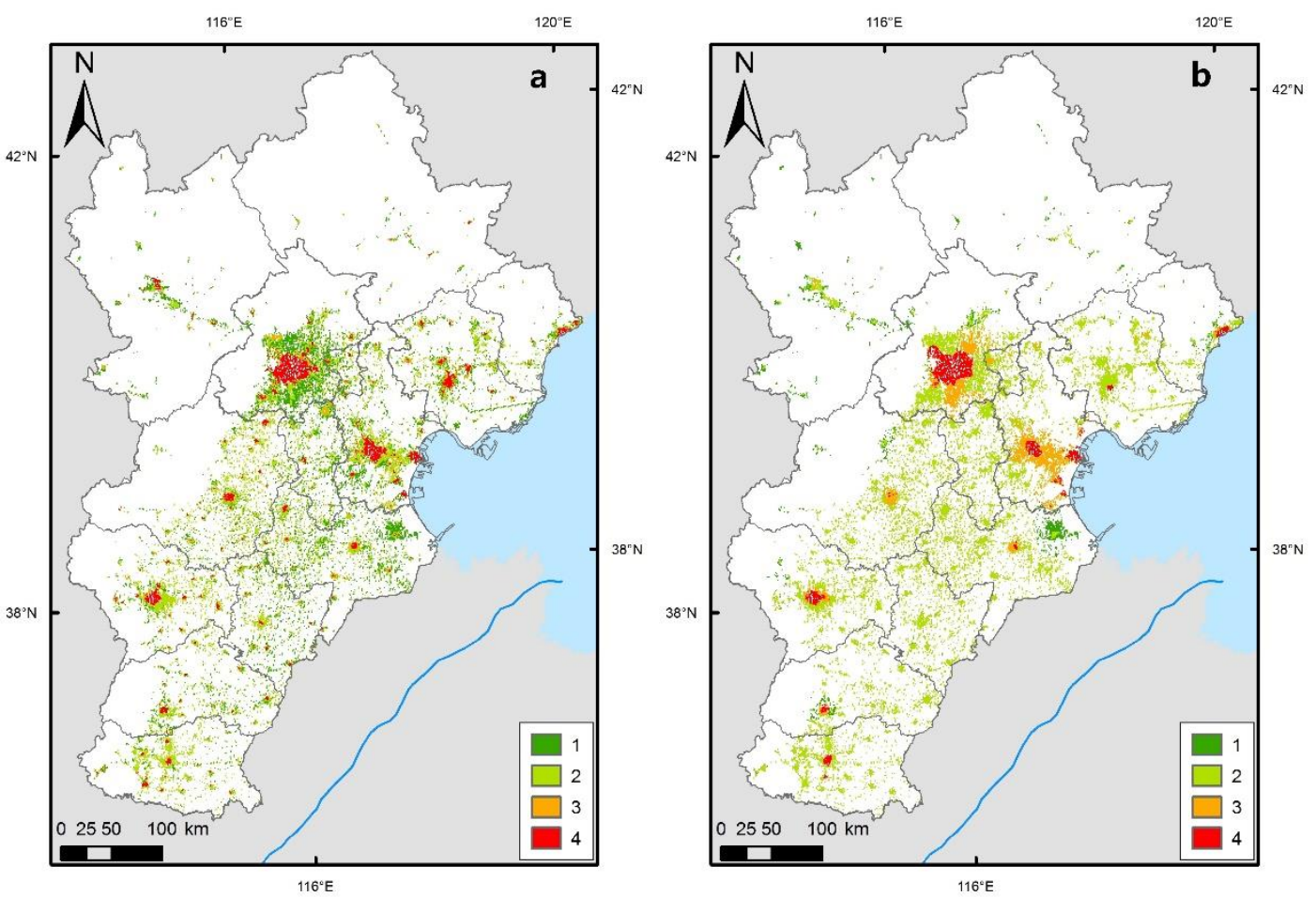

Figure 6 Exposure index for the BTH in (a) 1990 and (b) 2015. Numbers in legend correspond to exposure level.

\subsection{Vulnerability index}

The waterlogging vulnerability index in the built-up areas of the BTH comprehensively considered drainage pipe network density, vegetation coverage, and river network density. Since these factors have not changed significantly in recent decades, we used the 2015 value or the urban waterlogging vulnerability assessment.

According to the density of the drainage pipe network in the built-up areas of the $\mathrm{BTH}$, the drainage capacity was analyzed. The results showed that the drainage capacities of Beijing, Baoding, Zhangjiakou, Qinhuangdao, and other places were at Level-1 risk, the drainage capacity of Tianjin was at Level-4 risk, and the drainage capacities of other cities were between Level-2 and Level-3 (Figure 7a). The urban vegetation coverage rate for all 13 cities in BTH had Level-1 risk, and that of the surrounding area was between Level-1 to Level-3 (Figure 7b). The density of river 
network in BTH urban built-up area at $1 \mathrm{~km}$ grid demonstrated that the majority of the cities were at Level-1, while some areas of Tianjin, Cangzhou, featured Level-4 (Figure $7 \mathrm{c})$.
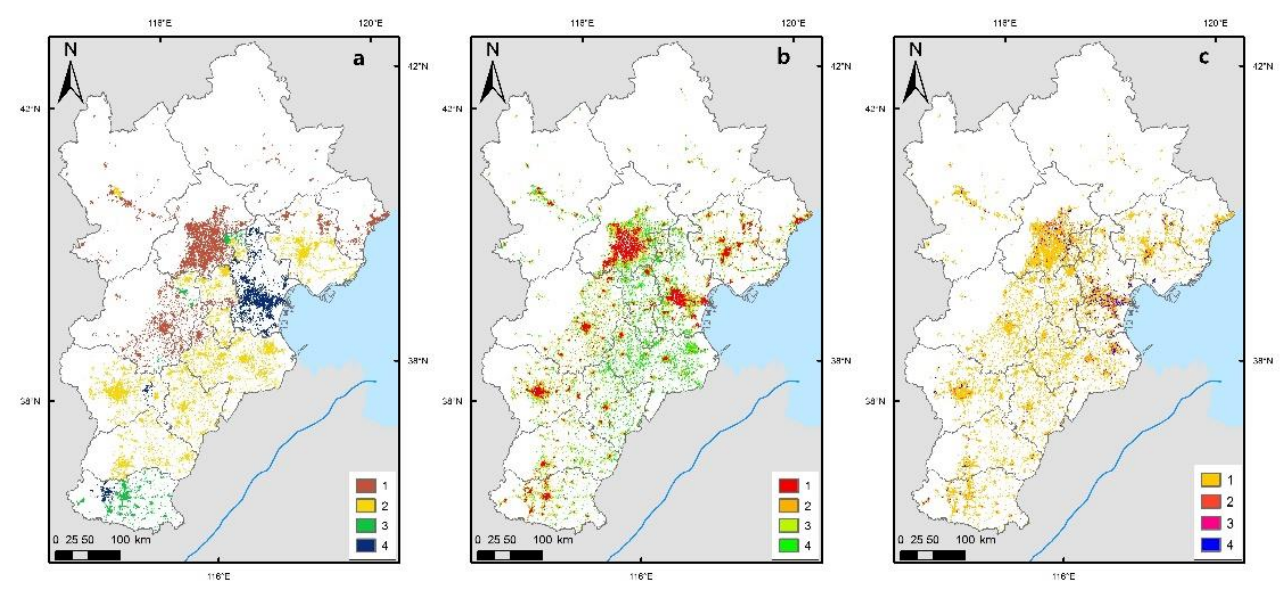

Figure 7 Urban drainage capacity (a), vegetation coverage (b), and river network density (c) grade in the BTH in 2015. Numbers in legend correspond to adaptability level.

The urban waterlogging adaptability index in the BTH was calculated using (5). As can be seen from Figure 8, the higher adaptability of the area resulted in lower waterlogging vulnerability. More specifically, Tianjin city had the highest waterlogging adaptability with Level-3 and above, reaching 99.9\% for all areas. Handan city waterlogging adaptability went second, between third to fourth grade with $63.4 \%$ for all areas (Figure 8). Most of the other cities had lower adaptability to waterlogging, mostly between first to second grade. In average, for the adaptability index by city, Tianjin had the highest score, followed by Handan, while Qinhuangdao had the lowest, followed by Beijing (Table 1). 


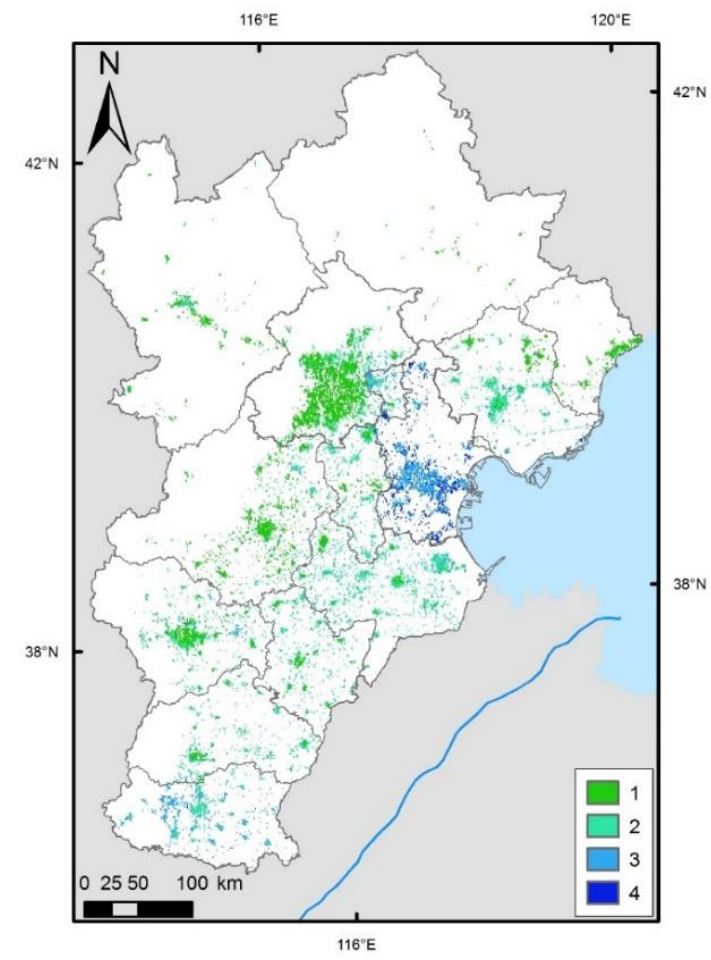

302 Figure 8 Adaptability to waterlogging risk in BTH. The data is for 2015 and is provided by the city.

303 Numbers in legend correspond to adaptability level.

304

305 306

Table 1 Hazard, exposure, adaptation, and risk indices for major in the BTH during 1961-1990 and 1991-2019 time periods.

\begin{tabular}{|c|c|c|c|c|c|c|c|}
\hline \multirow[t]{2}{*}{ Indices } & \multicolumn{3}{|c|}{ 1961-1990 } & \multicolumn{3}{|c|}{ 1991-2019 } & \multirow{2}{*}{$\frac{2015}{\text { Adaptation }}$} \\
\hline & Hazard & Exposure & Risk & Hazard & Exposure & Risk & \\
\hline Beijing & 1.95 & 2.02 & 2.46 & 2.75 & 2.67 & 2.67 & 1.76 \\
\hline Tianjin & 3.29 & 2.10 & 1.99 & 3.14 & 2.69 & 1.90 & 4.00 \\
\hline Shijiazhuang & 2.12 & 2.16 & 2.02 & 3.01 & 2.21 & 2.15 & 2.27 \\
\hline Tangshan & 3.78 & 1.94 & 2.54 & 2.70 & 2.03 & 1.87 & 2.45 \\
\hline Qinhuangdao & 3.99 & 2.06 & 2.75 & 3.43 & 2.10 & 2.44 & 1.56 \\
\hline Handan & 2.64 & 2.01 & 1.76 & 2.88 & 2.10 & 1.93 & 3.14 \\
\hline Xintai & 2.65 & 1.85 & 1.84 & 2.85 & 2.02 & 1.90 & 2.42 \\
\hline Baoding & 2.20 & 1.96 & 1.88 & 2.25 & 2.01 & 1.84 & 2.00 \\
\hline Zhangjiakou & 1.01 & 1.61 & 1.04 & 1.04 & 1.40 & 1.00 & 1.90 \\
\hline Chengde & 1.50 & 1.83 & 1.08 & 1.43 & 1.33 & 1.19 & 1.87 \\
\hline Cangzhou & 2.94 & 1.61 & 1.79 & 3.21 & 1.90 & 1.82 & 2.69 \\
\hline Langfang & 2.44 & 1.64 & 1.49 & 3.29 & 2.04 & 2.01 & 2.74 \\
\hline Hengshui & 3.02 & 1.65 & 1.86 & 2.39 & 1.99 & 1.49 & 2.40 \\
\hline
\end{tabular}

\subsection{Waterlogging risk}

The BTH urban waterlogging risk index was calculated from (2) and included hazard, 

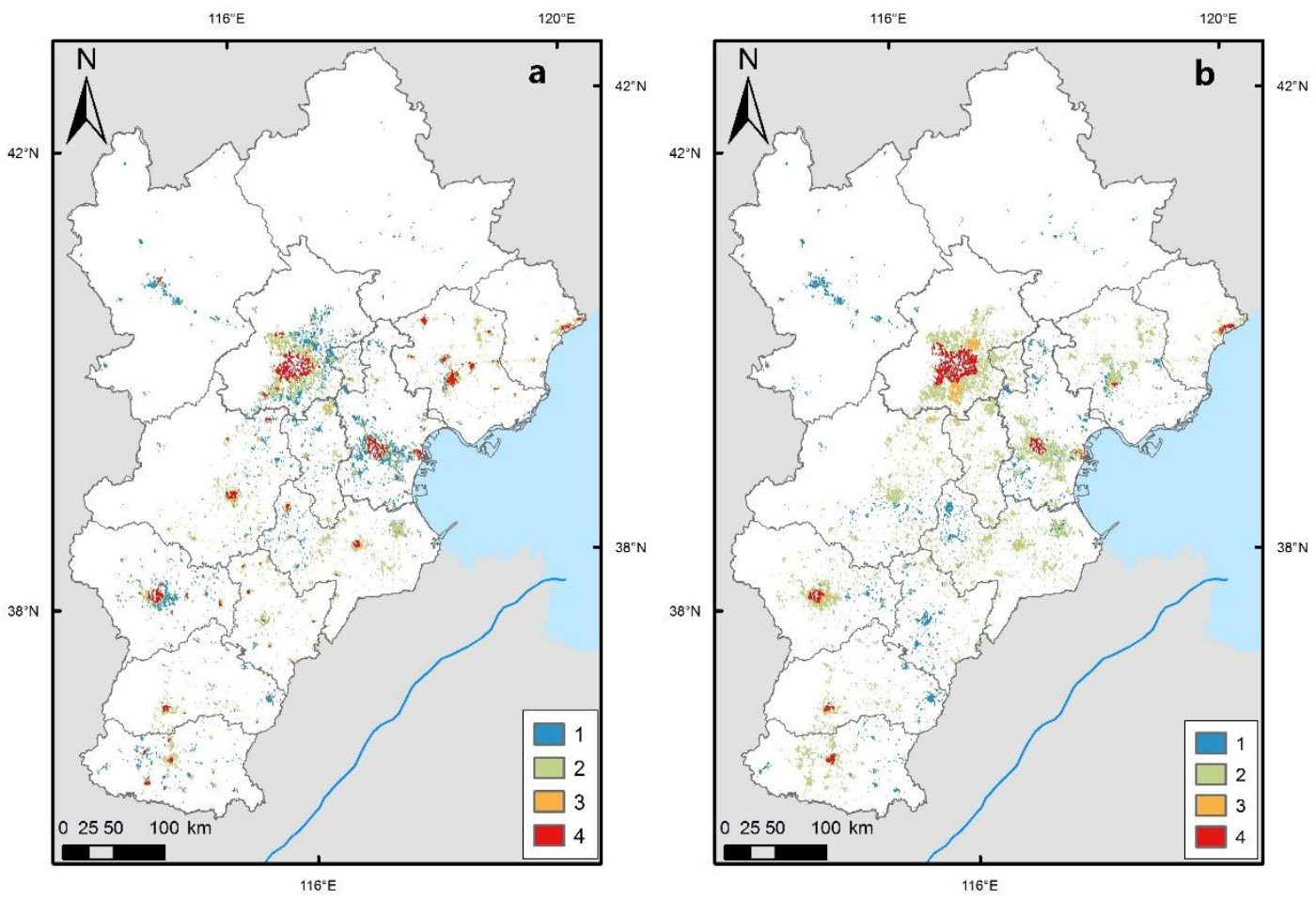

Figure 9 Urban waterlogging risk levels in the BTH for the time periods of (a) 1961-1990 and (b) 1991-2019. Numbers in legend correspond to waterlogging risk level.

The average waterlogging risk index for each city demonstrated that Qinhuangdao had the highest waterlogging risk for the years 1961-1990 (2.75), followed by Tangshan (2.54) and Beijing (2.46). Moreover, Beijing had the highest waterlogging risk for the 1991-2019 time period (2.67), followed by Qinhuangdao (2.44) and Shijiazhuang. (2.15). At the same time, Zhangjiakou, Chengde, and Hengshui had the lowest risks for both time periods (Table 1). The statistical analysis revealed that compared to the previous 30 years, except for Zhangjiakou, Tangshan, Chengde, and Hengshui, the areas at Level-1 waterlogging risk in other cities have decreased, and the total area at Level1 risk of BTH have decreased by 50\% (Table 2). Except for Zhangjiakou, Chengde, and Hengshui, the area at Level-2 risk in other cities had increased, and the total area at Level-2 has increased by nearly 55\%. Although the areas at Level-3 risk and above have been reduced by $17 \%$, the waterlogging risk varied greatly among cities, while Beijing 
had the largest increase in the area at Level-3 and above, accounting for $76 \%$. The areas at Level-4 risk increased most significantly in Beijing and Handan (by 52\% and 51\%, respectively). From 1991 to 2019, the areas at Level-3 risk and above in the BTH were mainly found in Beijing, Shijiazhuang, Tianjin, Qinhuangdao, Handan, and Xingtai, while from 1961 to 1990, they were mainly concentrated in Beijing, Tangshan, Tianjin, Shijiazhuang, Baoding, and Cangzhou.

Table 2. The area $\left(\mathrm{km}^{2}\right)$ of different waterlogging risk levels in the built-up areas of BTH cities for 1961-1990 and 1991-2019 time periods.

\begin{tabular}{ccccccccc}
\hline Period & \multicolumn{7}{c}{$1961-1990$} & \multicolumn{5}{c}{$1991-2019$} \\
\hline Risk level & 1 & 2 & 3 & 4 & 1 & 2 & 3 & 4 \\
& (Low) & (Moderate) & (High) & (Very High) & (Low) & (Moderate) & (High) & (Very High) \\
\hline Beijing & 795 & 1225 & 126 & 515 & 31 & 1500 & 347 & 783 \\
Tianjin & 955 & 405 & 73 & 233 & 420 & 1060 & 52 & 134 \\
Shijiazhuang & 461 & 270 & 33 & 149 & 136 & 586 & 88 & 103 \\
Tangshan & 165 & 471 & 137 & 222 & 177 & 787 & 12 & 19 \\
Qinhuangdao & 33 & 148 & 43 & 63 & 2 & 203 & 19 & 63 \\
Handan & 343 & 315 & 39 & 35 & 159 & 505 & 15 & 53 \\
Xintai & 228 & 257 & 35 & 53 & 154 & 354 & 26 & 39 \\
Baoding & 399 & 468 & 62 & 87 & 190 & 805 & 21 & 0 \\
Zhangjiakou & 365 & 15 & 6 & 16 & 402 & 0 & 0 & 0 \\
Chengde & 48 & 13 & 5 & 7 & 70 & 3 & 0 & 0 \\
Cangzhou & 406 & 852 & 51 & 76 & 276 & 1085 & 24 & 0 \\
Langfang & 398 & 329 & 44 & 6 & 7 & 752 & 18 & 0 \\
Hengshui & 142 & 333 & 25 & 37 & 289 & 248 & 0 & 0 \\
\hline
\end{tabular}

The changes in waterlogging risks for BTH cities were analyzed in the context of global climate change and urbanization. It revealed that the risk of waterlogging for Beijing had increased the most. More specifically, comparing the time periods from 1961-1990 and from 1991 to 2019, the areas at Level-1 waterlogging risk in Beijing decreased from $29.8 \%$ to $1.1 \%$, and that at Level-2, Level-3 and Level-4 risks increased from $46.0 \%, 4.7 \%$, and $19.4 \%$ to $56.3 \%, 13.0 \%$ and $29.4 \%$, respectively. In the past 30 years, areas in Beijing at Level-4 waterlogging risk were mostly found in Haidian, Chaoyang, Dongcheng, and Xicheng districts. The latter was mainly due to the high 
hazard and exposure (Figure 10). Compared to the time period from 1961 to 1990, the waterlogging risks in Shunyi, Tongzhou, Daxing, and Fangshan districts have increased. These districts were the fastest developing districts in Beijing over the past 10 years, with the exposure index changed from Level-2 to Level-3 or Level-4, and the hazard also increased from Level-1 and Level-2 to Level-3 and Level-4.
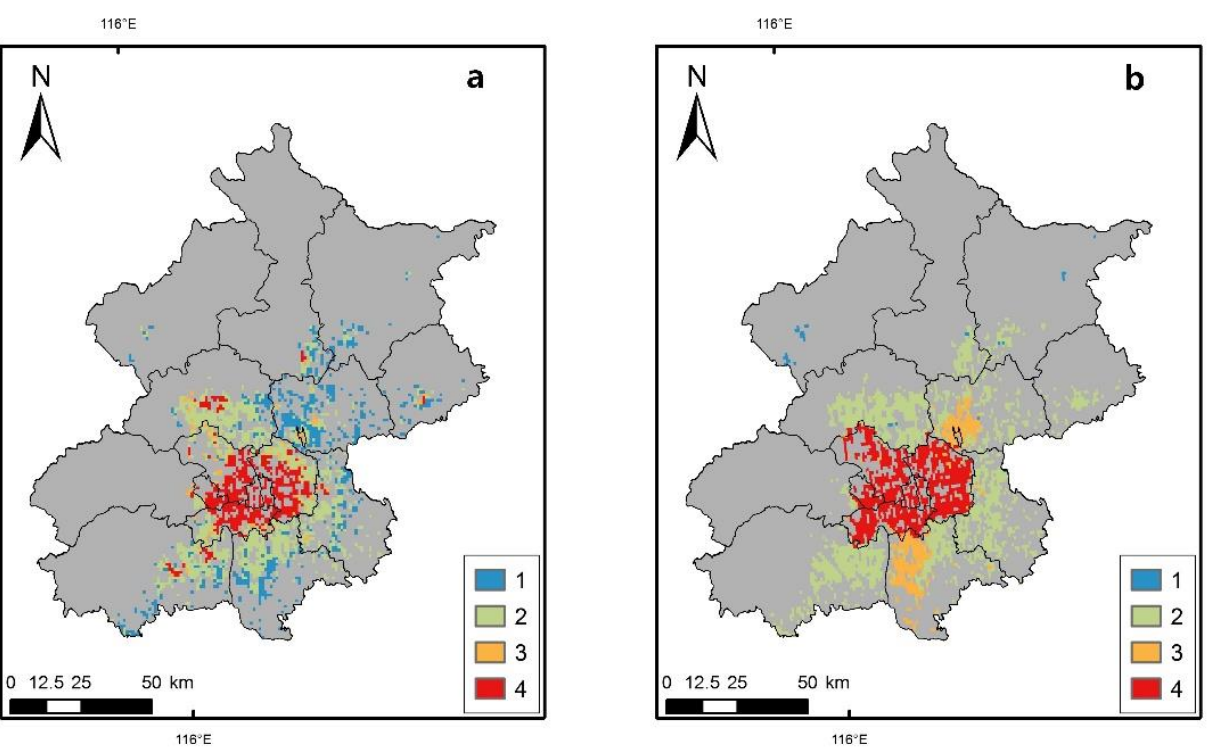

Figure 10 Waterlogging risk levels in various districts in Beijing for (a) 1961-1990 and (b) 19912019 time periods.

\section{Conclusion and discussion}

We have investigated the combined influence of global climate change and urbanization on 13 cities in Beijing, Tianjin, and Hebei provinces for the past 60 years. We have found that Beijing, Tianjin, Shijiazhuang, Qinhuangdao, Handan, and Xingtai have become cities with the Level-3 and Level-4 waterlogging risks. Among them, Beijing has the highest risk of waterlogging. The areas at Level-3 and above have accounted for $62 \%$ of the total area of the BTH region and demonstrated an increase of $76 \%$. The areas at Level-4 risk have been mainly concentrated in major urban areas such as Haidian, Chaoyang, Dongcheng, and Xicheng districts. Compared to the 1961 to 1990 time period, the areas at Level-1 risk have decreased by nearly $50 \%$. In particular, the areas at Level-2 risk have increased by nearly $55 \%$ and that ones at Level- 
3 and above have decreased. However, the variations in each city were quite different. Among them, the areas at Level-4 risk have increased by $52 \%$ and $51 \%$ in Beijing and Handan, while that in Tangshan and Baoding have decreased significantly. In recent decades, the rapid urbanization process of the BTH region has changed its original natural landscape and ecosystem. With the fast-growing of economic and social exposure and the short-time rainfall intensity, the problem of urban waterlogging for the BTH urban agglomeration has become particularly important. In particular, the planning, design, safety operation, and maintenance of megacities have become very challenging. For example, the built-up area in Beijing has increased from $239 \mathrm{~km}^{2}$ in the early 1980 s to $1,400 \mathrm{~km}^{2}$ today (Tan et al., 2018), leading to the decrease of the density of natural river networks. At the same time, the newly built pipeline network has a low level of fortification that speeds up the surface convergence, causing cities being washed away. Additionally, Beijing's economic and social exposure has also increased sharply. In 2015, Beijing's total population reached 21.7 million, and its GDP reached 2.3 trillion yuan, twice and 45 times that of 1990. In addition, global climate change caused a dramatic increase in the 1-hour and 1-day rain intensity, leading to an increase of the waterlogging risk in Beijing. Therefore, it is necessary to implement the BTH coordinated development strategy and relieve Beijing's non-capital functions. In the future development plan of the BTH region, the changes in short-time precipitation intensity caused by climate change should be considered. Moreover, the infrastructure construction standards of urban drainage should be improved, the drainage pipeline networks should be carefully arranged. Moreover, the scientific approach to the utilization of vegetation coverage and improvement of the city's adaptability to extreme rainstorms are required. Finally, the enhancement of urban resilience and reduction of the waterlogging risks caused by rainstorms should also be done.

In this paper, a quantitative assessment of the urban waterlogging risk in the BTH region was conducted at the kilometer-grid scale, considering hazard, exposure, and vulnerability factors. However, the formation mechanism of urban waterlogging is complex, especially in the context of global climate change. The impact of urbanization on the hazard of waterlogging needs to be further studied. Finally, this study has several 
398 limitations. In particular, the density of urban drainage network can only be assigned to 399 grid points belonging to the same administrative area through statistical yearbook data 400 and the actual drainage capacity of each network point, the impact on terrain have not 401 been considered.

402

403 Conflict of Interest: The authors declared that we do not have any commercial or 404 associative interest that represents a conflict of interest in connection with the work 405 submitted.

406 Funding Statement: This work was supported by the National Key R\&D Program of 407 China (Grant No. 2018YFA0606302).

408 Author's Contribution: Yujie Wang contributed to this paper by performing analyses 409 and drafting the paper. Jianqing Zhai performed analyses by calculating the hazard 410 factors, exposure indicators, adaptability and risk level to heavy rainfall and 411 waterlogging in the BTH urban agglomeration. Lianchun Song conceived the study and 412 modified the complete research and manuscript. 


\section{References}

Bai X, Dawson RJ, Rgevorsatz D (2018) Six research priorities for cities and climate change. Nature, $555,23-25$.

Chen ZS, Liu ZM, Lu JF (2010) Comparative analysis of parameter estimation methods of generalized extreme value distribution. Acta Scientiarum Natralium Universitatis Sunyatseni 49:105-109

Fu JY, Jiang D, Huang YH (2014) China kilometer grid population distribution data set. Acta geographica Sinica. 69(supplement):41-44

Gu XH, Zhang Q, Vijay PS, et al. (2019) Potential contributions of climate change and urbanization to precipitation trends across China at national, regional and local scales. Int J Climatol, 39(6): 2998-3012.

Hao L, Min J, Ding YH (2011) Analysis of precipitation events changes and causes for rainstorm events reduction in North China, Chin. J. Geophys., 54(5), 1160-1167

Hu HB (2016) Rainstorm flash flood risk assessment using genetic programming: a case study of risk zoning in Beijing. Nat. Hazards 83: 485-500

Kaźmierczak A, Cavan G (2011) Surface water flooding risk to urban communities: Analysis of vulnerability, hazard and exposure. Landscape Urban Plan, 103: 185-197

Kharin VV, Zwiers FW, Zhang X, Wehner M (2013). Changes in temperature and precipitation extremes in the CMIP5 ensemble. Climatic change, 119(2), 345-357.

Li N, Qiu JW, Cheng XT, Lu JK (2002) Study on simulation system of rainstorm waterlogging in Tianjin City. J. of Nat. Disasters. 11(2): 112-118

Li SY, J. Ma JJ (2011) Impact of urbanization on precipitation in Beijing area, J. Meteorol. Sci., $31(4), 414-421$.

Li Z, Yan ZW, Tu K, et al. (2015) Changes of precipitation and extremes and the possible effect of urbanization in the Beijing metropolitan region during 1960-2012 based on homogenized observations. Adv Atmos Sci, 32(9): 1173-1185.

Liang P, Ding YH (2017) The long-term variation of extreme heavy precipitation and its link to urbanization effects in Shanghai during 1916-2014. Adv Atmos Sci, 34: 321-334

Liang SJ, Cheng SJ, Hao LS, et al. (2018) Analysis on the characteristics of hourly precipitation variations in Beijing-Tianjin-Hebei region during 1970-2015. Torrential Rain and Disasters, 
37(2): 105-114

National Climate Center (NCC) (2013) China Climate Bulletin 2012, 158PP

Ren, Z. H., Yu, Y., Zhou, F. L., et al., 2012. Quality Detection of Surface Historical Basic Meteorological Data. Journal of Applied Meteorological Science, 23, 739-747.

Saeed G, Bahram S, Reza, M (2010) Derivation of probabilistic thresholds of spatially distributed rainfall for flood forecasting. Water Resour. Manag. 24: 3547-3559

Shi Y, Han ZY, Xu Y, et al (2019) Future changes of climate extremes in Xiongan New Area and Jing-Jin-Ji district based on high resolution $(6.25 \mathrm{~km})$ combined statistical and dynamical downscaling datasets. Climate Change Research, 2019, 15 (2): 140-149

Sillmann J, Kharin VV, Zhang X, Zwiers FW et al (2013). Climate extremes indices in the CMIP5 multimodel ensemble: Part 1. Model evaluation in the present climate. Journal of Geophysical Research: Atmospheres, 118(4), 1716-1733.

Song X, Zhang J, AghaKouchak A, Roy SS, et al. (2014) Rapid urbanization and changes in spatiotemporal characteristicsof precipitation in Beijing metropolitan area, J. Geophys. Res. Atmos., 119: 11,250-11,271

Sun S., Zhai JQ., Li Y., Huang DP et al (2020). Urban waterlogging risk assessment in welldeveloped region of Eastern China. Physics and Chemistry of the Earth, 115, 102824

Tan C, Kong F, Guo J, et al (2018) Spatial and temporal patterns of heavy rainfall in different urbanized areas of China from 1961 to 2014: Taking Beijing-Tianjin-Hebei, Yangtze River Delta and Pearl River Delta as an example. Journal of Catastrophology, 33 (3) : 132-139

Wang C, Wu J, Wang X, He X (2018) Application of the hidden Markov model in a dynamic risk assessment of rainstorms in Dalian, China. Stoch. Environ. Res. Risk Assess. 32: 2045-2056

Wang XF, Wang S, Ren L, Zeng ZZ (2017) Spatial distribution of rainstorm hazard risk based on EW-AHP in mountainous scenic area of China. Hum. Ecol. Risk Assess. 23, 925-943

Wang Y, Gao C, Wang A, et al. (2014) Temporal and spatial variation of exposure and vulnerability of flood disaster in China. Clim Change Res, 6: 391-398

Wang YJ, Song LC, Han ZY, et al. (2020) Climate-related risks in the construction of Xiongan New Area, China. Theoretical and Applied Climatology, 141:1301-1311.

Xie WS, Wu R, Ding XJ (2018) Risk assessment and early warning of urban waterlogging based on FloodArea model. Resources and Environment in the Yangtze Basin. 27(12):2848-2855 
Yang P, Ren GY, Yan PC (2017) Evidence for a Strong Association of Short-Duration Intense Rainfall with Urbanization in the Beijing Urban Area. J Climate, 30(15): 5851-5870.

Yang L, Tian FQ, Smith J, et al. (2014) Urban signatures in the spatial clustering of summer heavy rainfall events over the Beijing metropolitan region. Journal of Geophysical ResearchAtmosphere, 119 (3): 1203-1217

Yin S, Li W, Chen D, Jeong JH, et al. (2011) Diurnal variations of summer precipitation in the Beijing area and the possible effect of topography and urbanization, Adv. Atmos. Sci., 28(4): $725-734$

Yu H, Zhao Y, Fu Y, Li L (2018a) Spatiotemporal variance assessment of urban rainstorm waterlogging affected by impervious surface expansion: a case study of Guangzhou, China. Sustainability 10, 3761

Yu R, Zhai PM, Lu YY (2018b) Implications of differential effects between 1.5 and $2{ }^{\circ} \mathrm{C}$ global warming on temperature and precipitation extremes in China's urban agglomerations. Int J Climatol, 38: 2374-2385

Zhang Y (2010) Heavy rainfall in the urban environment, Doctoral Thesis, Princeton University, New Jersey, USA.

Zhai PM, Zhang X, Wan H, et al. (2005) Trends in total precipitation and frequency of daily precipitation extremes over China. Journal of climate, 18(7): 1 096-1 108

Zhai PM, Yuan YF, Yu R, Guo JP (2018) Climate change and sustainable development for cities. Chin Sci Bull 64:1995-2001

Zhang CL, Chen F, Miao SG, et al (2009) Impacts of urban expansion and future green planting on summer precipitation in the Beijing metropolitan area. J. Geophys. Res., 114: D02116

Zhang Y, Smith JA, Luo L, Wang Z, et al (2014), Urbanization and rainfall variability in the Beijing metropolitan region, J. Hydrometeorol., 2219-2235

Zheng GG, Jiao MY, Ding YH, Song LC (2019) China Climate.China Meteorological Press, 318 pp.

Zheng Y, Zhai JQ, Wu ZY, et al (2018) A typology analysis on resilient cities based on adaptive cycle. China population, resources and environment, 28( 3) : 31-38

Zhou XY, Bai ZJ, Yang YH (2017) Linking trends in urban extreme rainfall to urban flooding in 29 China. Int J Climatol, 37(13):4586-4593. 


\section{Figures}

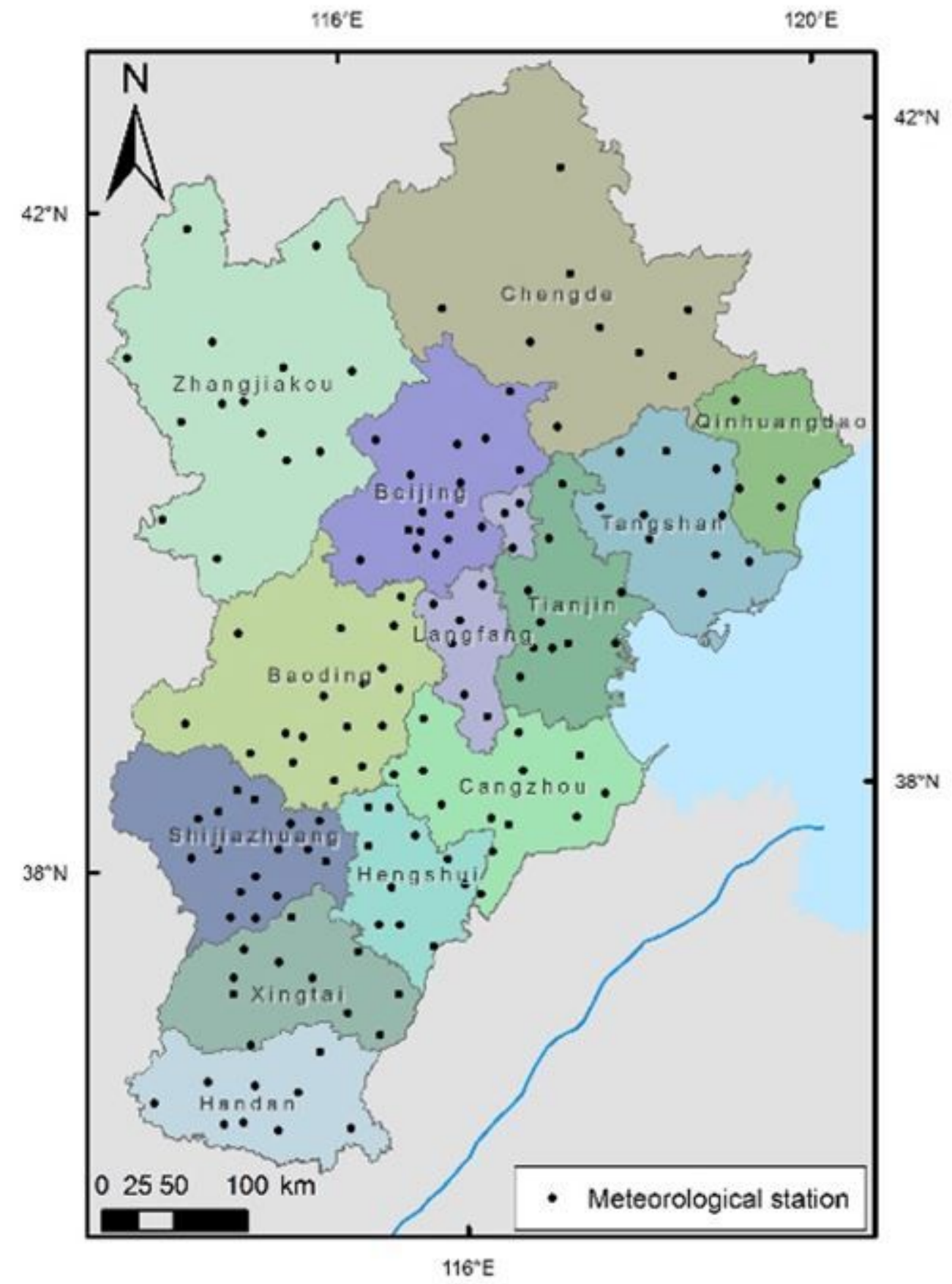

\section{Figure 1}

Administrative divisions and meteorological stations in the BTH. Note: The designations employed and the presentation of the material on this map do not imply the expression of any opinion whatsoever on the part of Research Square concerning the legal status of any country, territory, city or area or of its authorities, or concerning the delimitation of its frontiers or boundaries. This map has been provided by the authors. 

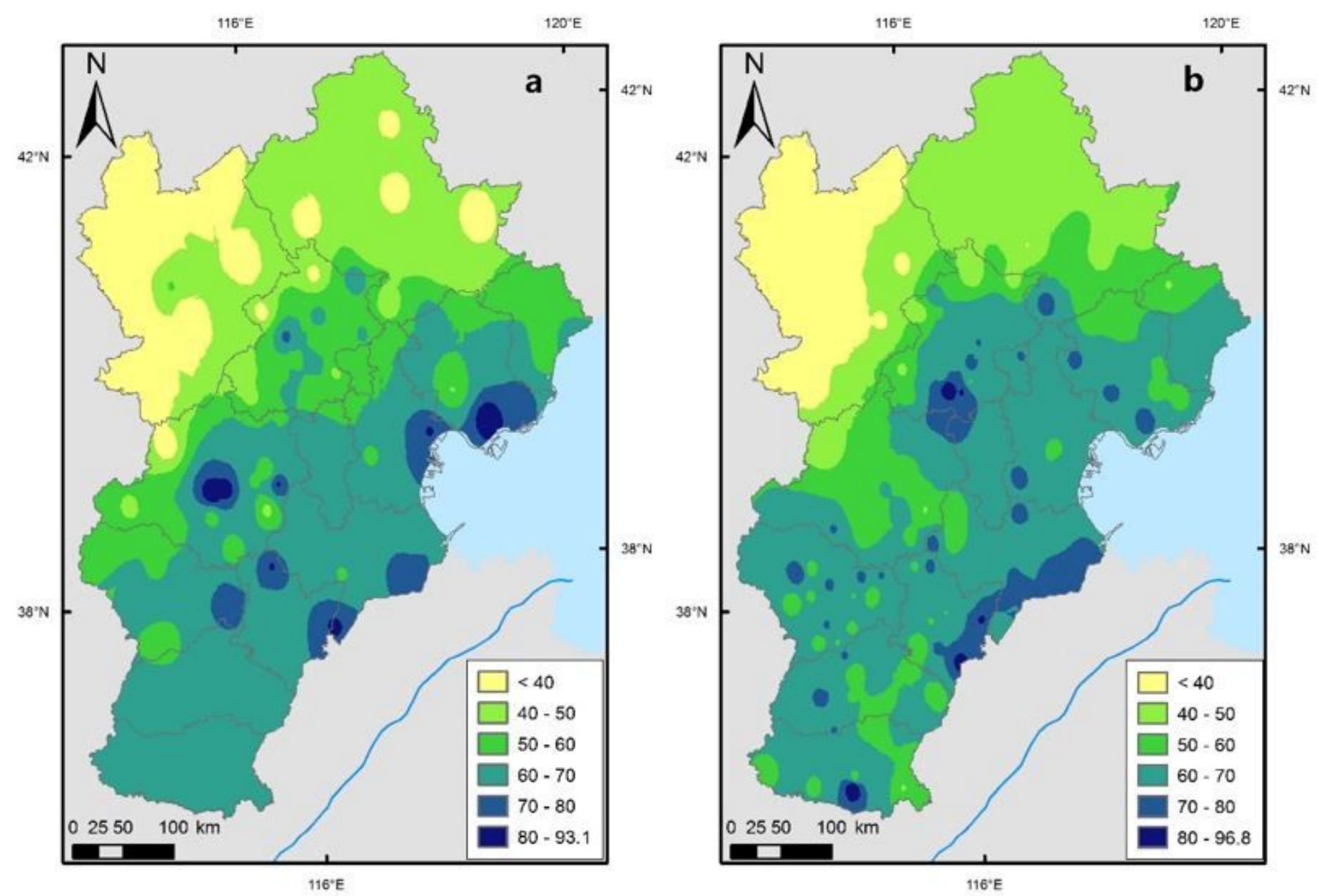

Figure 2

One-hour precipitation distribution in the BTH for (a) 1961-1990 and (b) 1991-2019 time periods. Note: The designations employed and the presentation of the material on this map do not imply the expression of any opinion whatsoever on the part of Research Square concerning the legal status of any country, territory, city or area or of its authorities, or concerning the delimitation of its frontiers or boundaries. This map has been provided by the authors. 

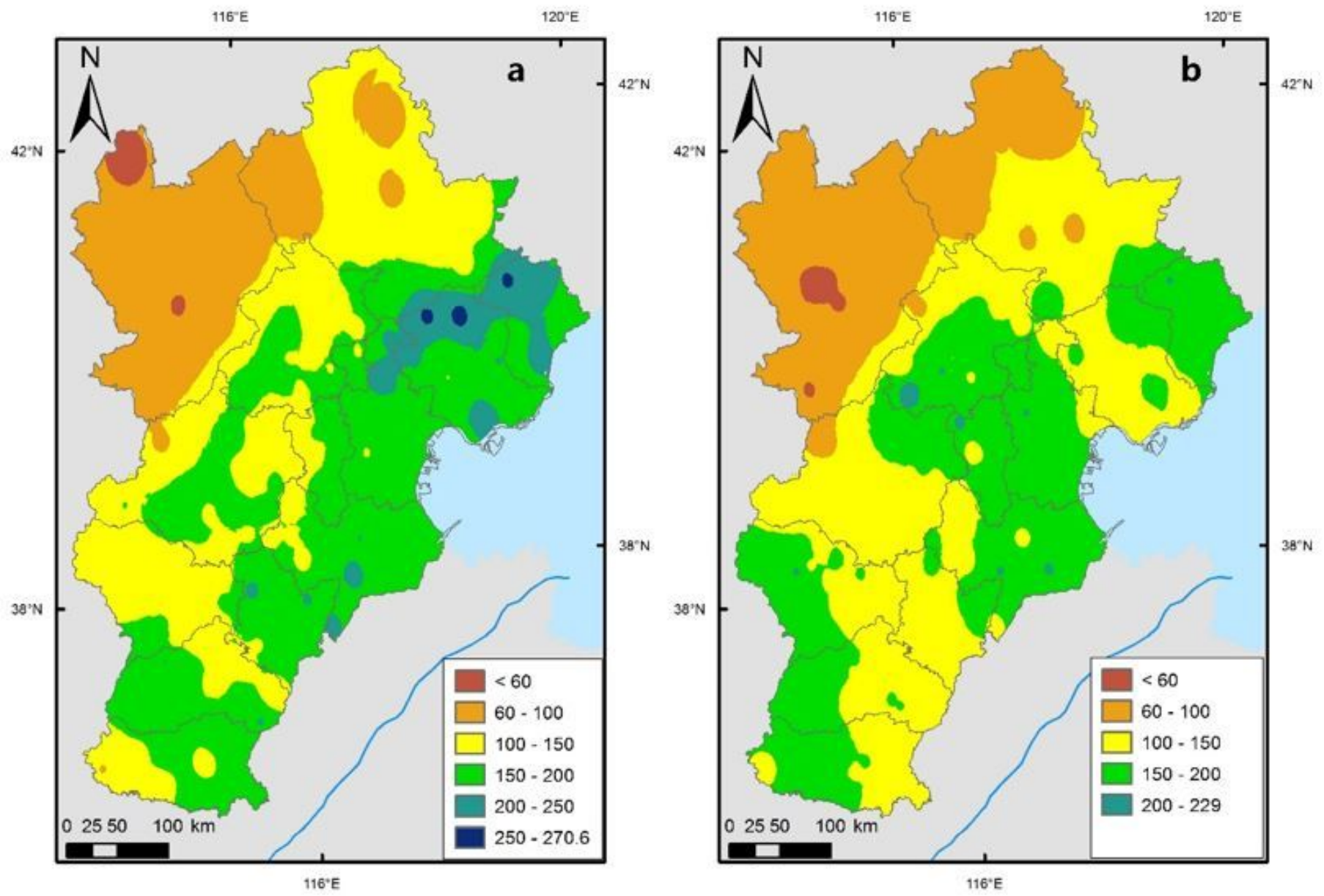

Figure 3

One-day precipitation distribution in the BTH for (a) 1961-1990 and (b) 1991-2019 time periods. Note: The designations employed and the presentation of the material on this map do not imply the expression of any opinion whatsoever on the part of Research Square concerning the legal status of any country, territory, city or area or of its authorities, or concerning the delimitation of its frontiers or boundaries. This map has been provided by the authors. 

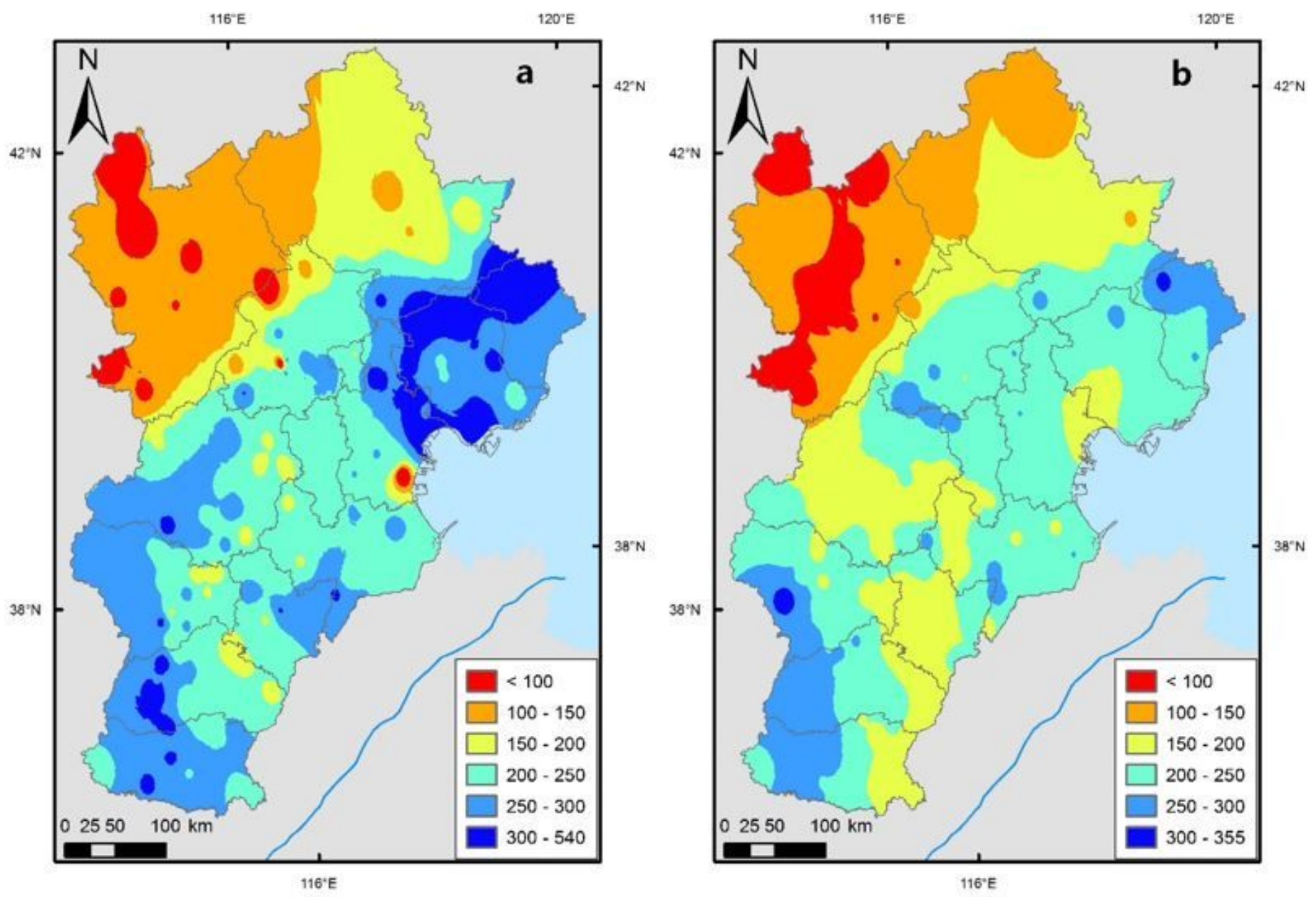

\section{Figure 4}

Five-day precipitation distribution in the BTH for (a) 1961-1990 and (b) 1991-2019 time periods. Note: The designations employed and the presentation of the material on this map do not imply the expression of any opinion whatsoever on the part of Research Square concerning the legal status of any country, territory, city or area or of its authorities, or concerning the delimitation of its frontiers or boundaries. This map has been provided by the authors. 

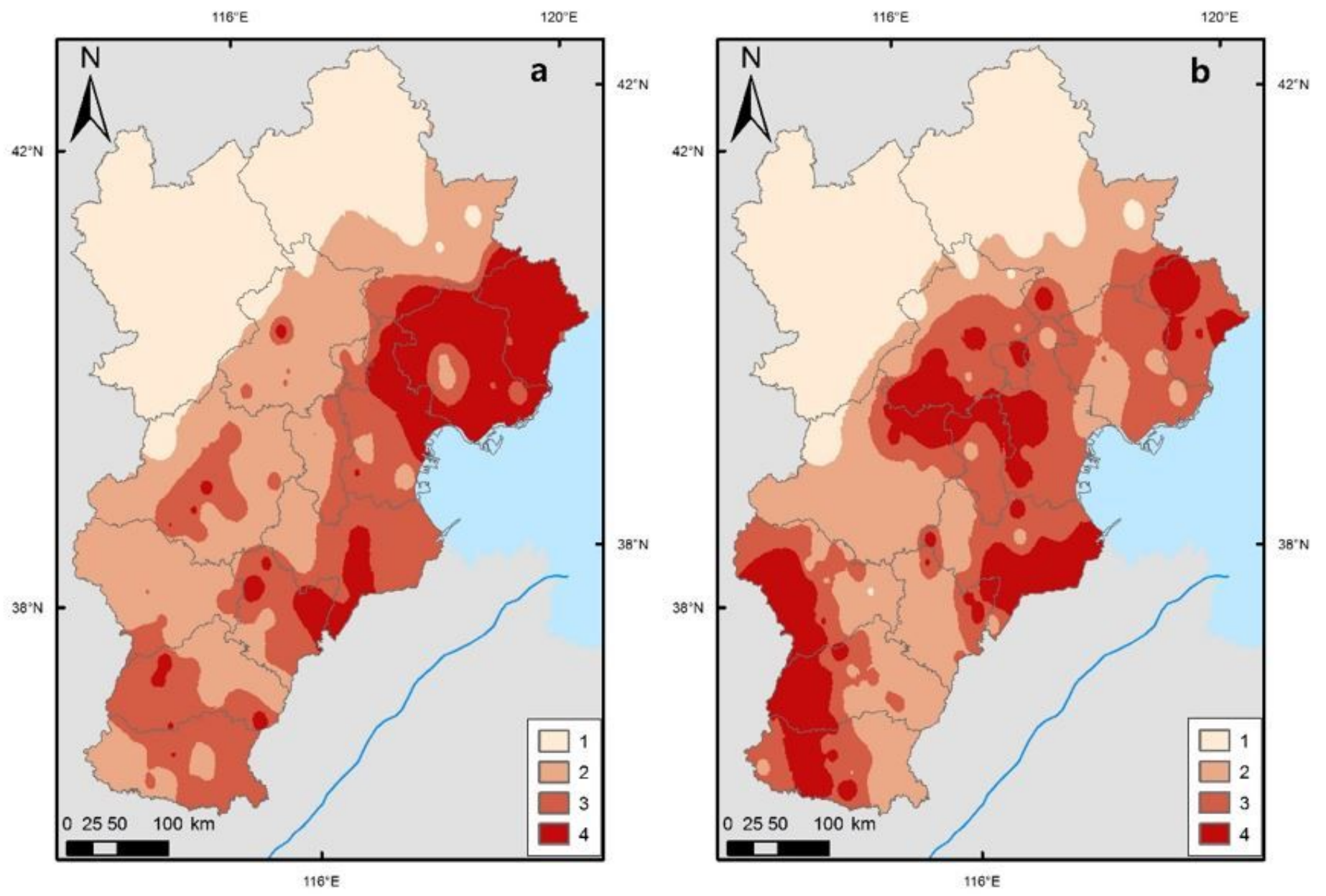

Figure 5

Hazard intensity for (a) 1961-1990 and (b) 1991-2019 time periods. It was measured once in every 20 years. Numbers is legend correspond to hazard intensity levels. Note: The designations employed and the presentation of the material on this map do not imply the expression of any opinion whatsoever on the part of Research Square concerning the legal status of any country, territory, city or area or of its authorities, or concerning the delimitation of its frontiers or boundaries. This map has been provided by the authors. 

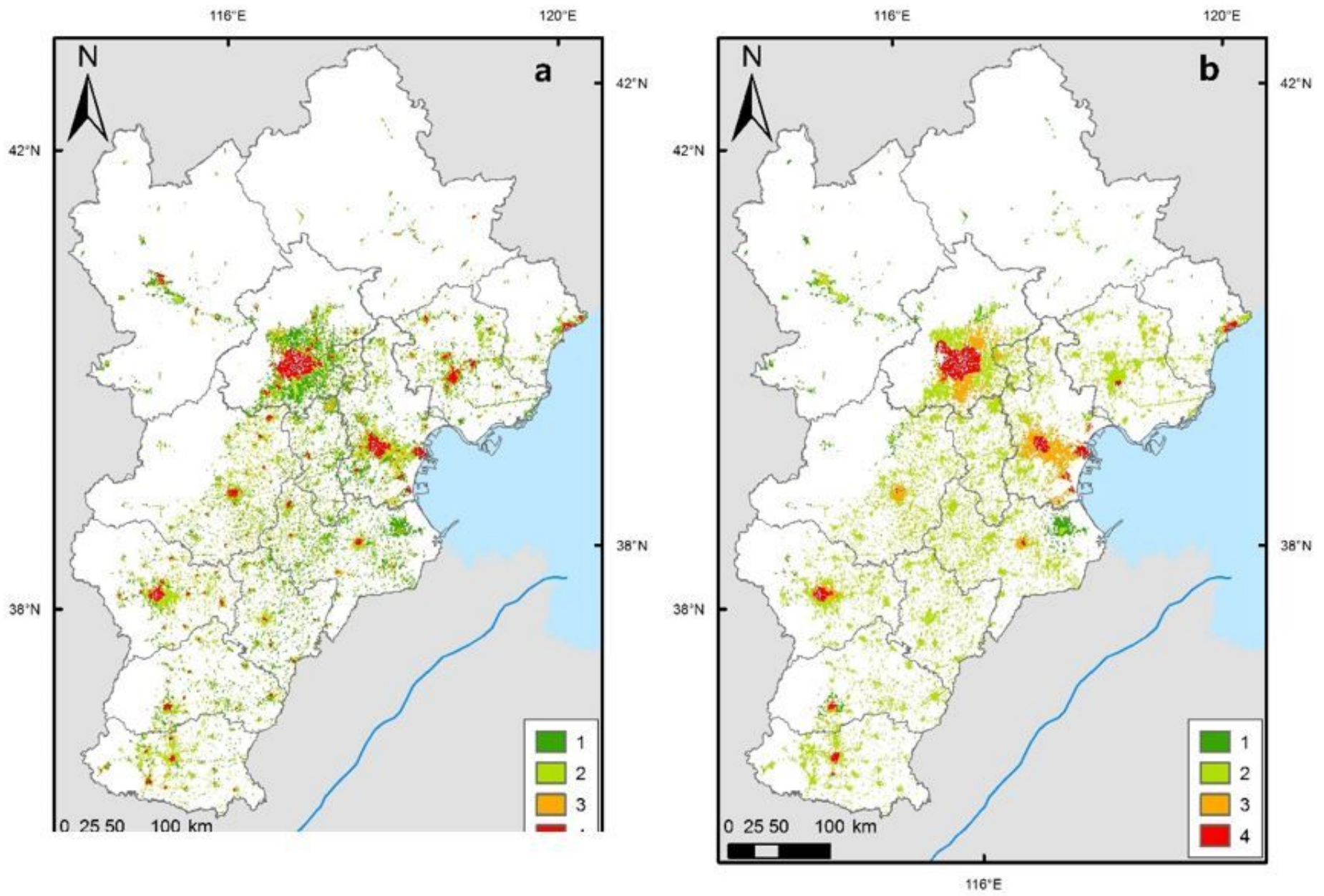

\section{Figure 6}

Exposure index for the BTH in (a) 1990 and (b) 2015. Numbers in legend correspond to exposure level. Note: The designations employed and the presentation of the material on this map do not imply the expression of any opinion whatsoever on the part of Research Square concerning the legal status of any country, territory, city or area or of its authorities, or concerning the delimitation of its frontiers or boundaries. This map has been provided by the authors. 

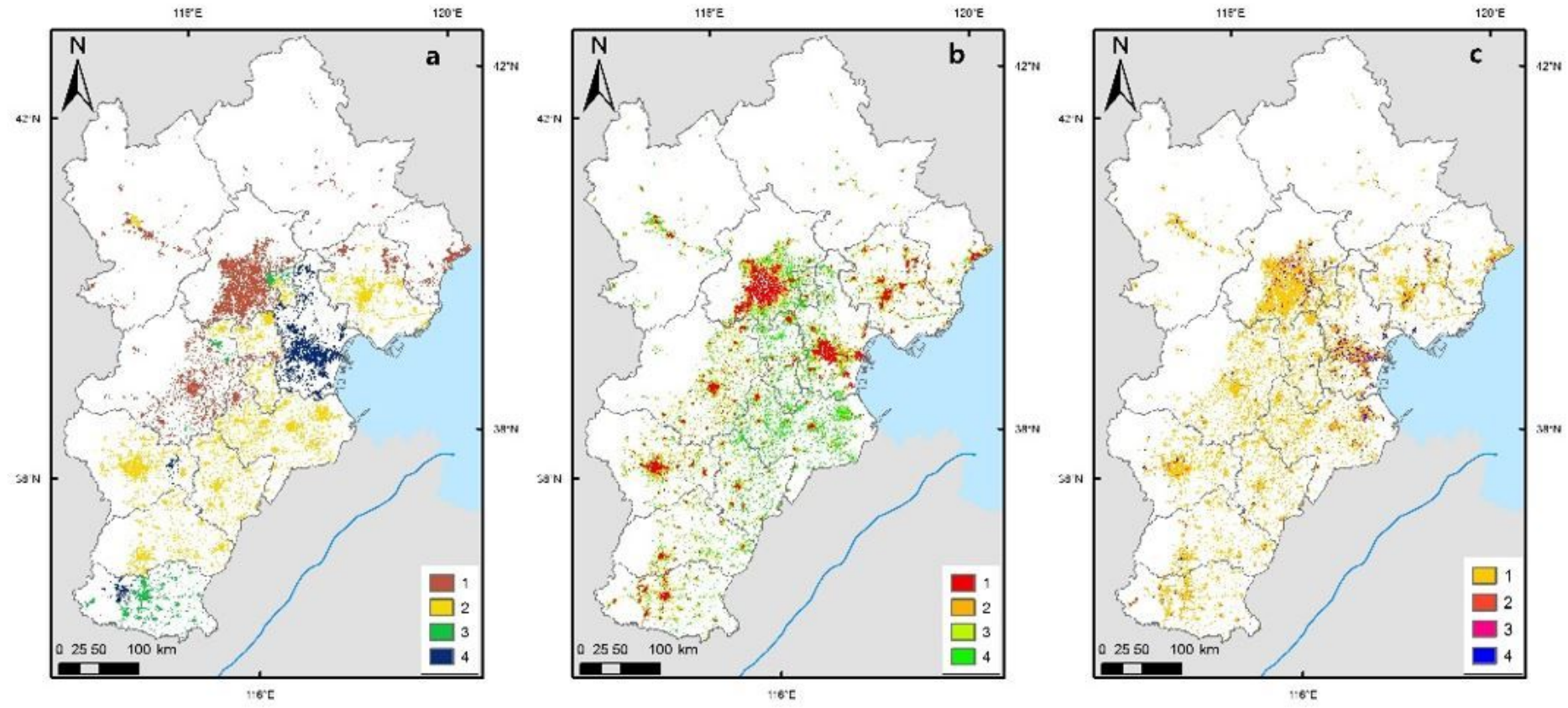

Figure 7

Urban drainage capacity (a), vegetation coverage (b), and river network density (c) grade in the BTH in 2015. Numbers in legend correspond to adaptability level. Note: The designations employed and the presentation of the material on this map do not imply the expression of any opinion whatsoever on the part of Research Square concerning the legal status of any country, territory, city or area or of its authorities, or concerning the delimitation of its frontiers or boundaries. This map has been provided by the authors. 


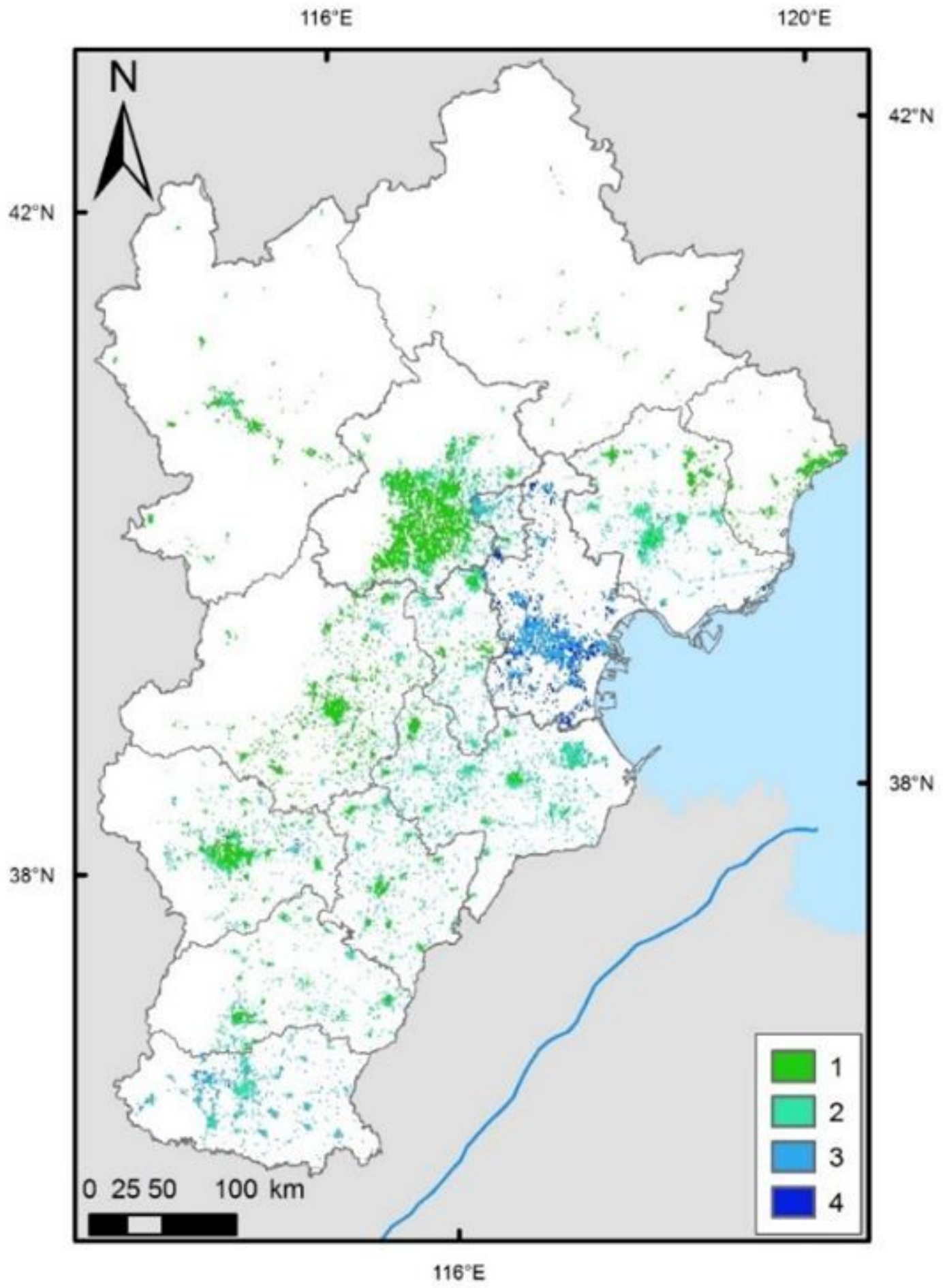

\section{Figure 8}

Adaptability to waterlogging risk in BTH. The data is for 2015 and is provided by the city. Numbers in legend correspond to adaptability level. Note: The designations employed and the presentation of the material on this map do not imply the expression of any opinion whatsoever on the part of Research Square concerning the legal status of any country, territory, city or area or of its authorities, or concerning the delimitation of its frontiers or boundaries. This map has been provided by the authors. 

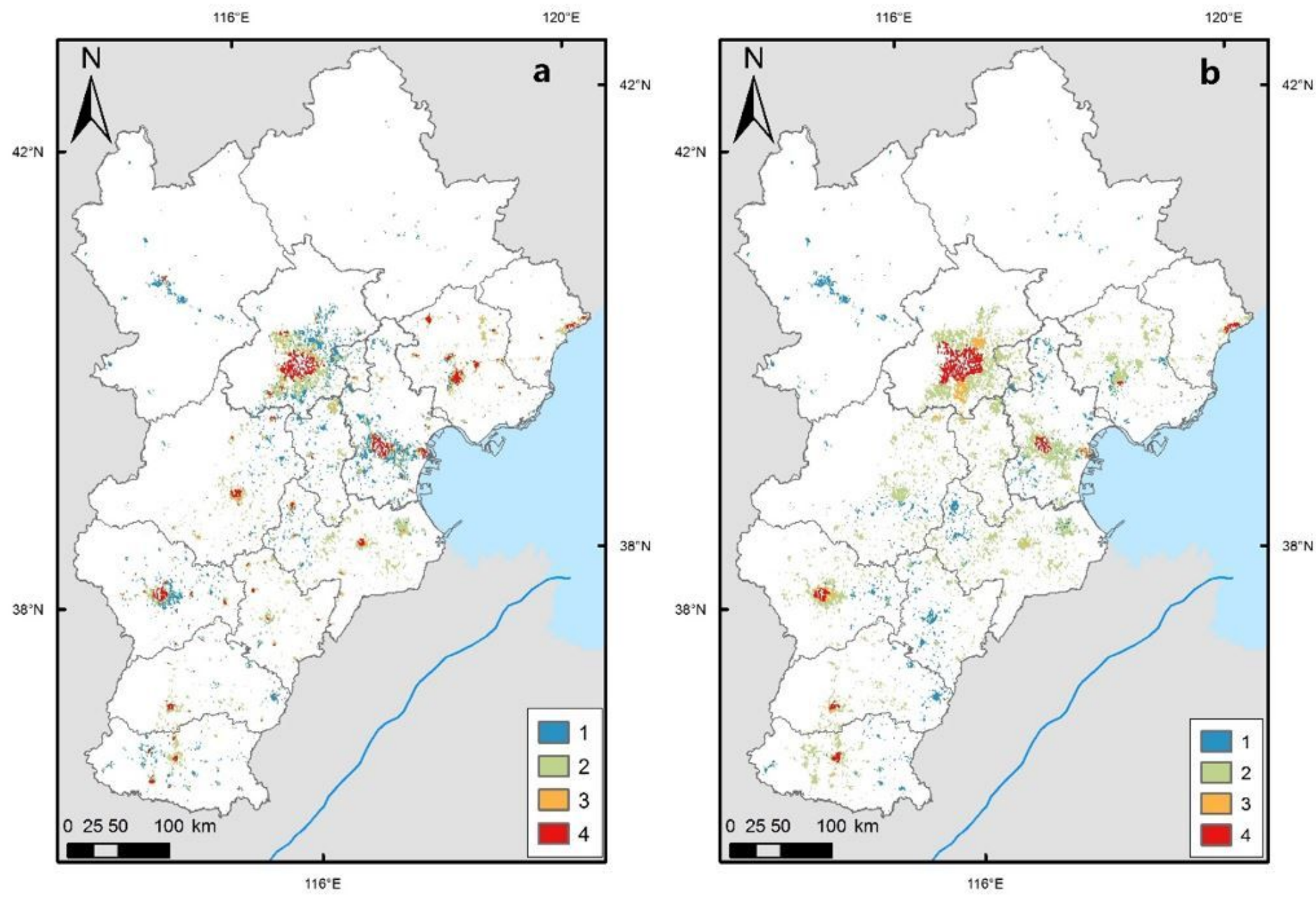

Figure 9

Urban waterlogging risk levels in the BTH for the time periods of (a) 1961-1990 and (b) 1991-2019. Numbers in legend correspond to waterlogging risk level. Note: The designations employed and the presentation of the material on this map do not imply the expression of any opinion whatsoever on the part of Research Square concerning the legal status of any country, territory, city or area or of its authorities, or concerning the delimitation of its frontiers or boundaries. This map has been provided by the authors. 

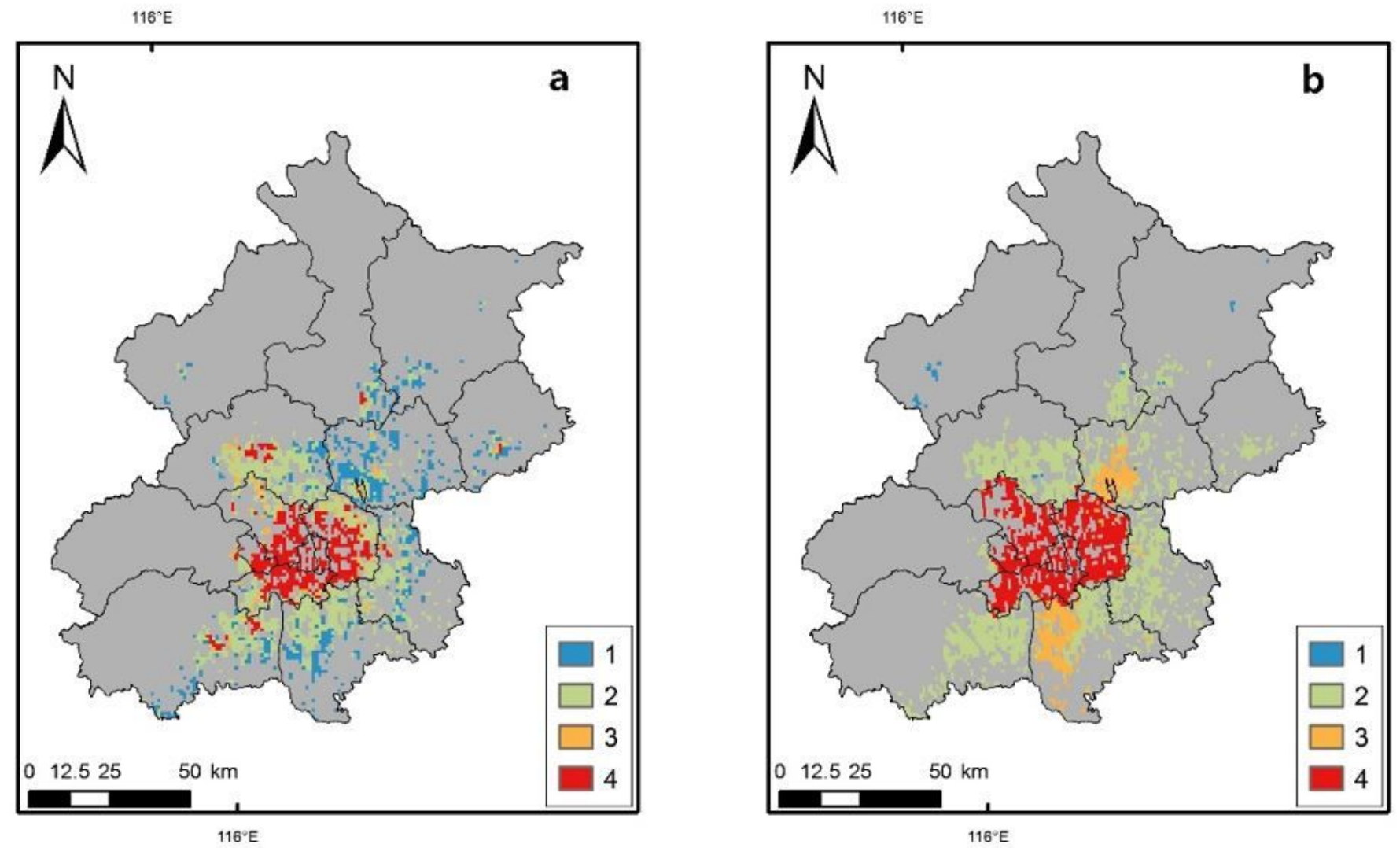

Figure 10

Waterlogging risk levels in various districts in Beijing for (a) 1961-1990 and (b) 1991-2019 time periods. Note: The designations employed and the presentation of the material on this map do not imply the expression of any opinion whatsoever on the part of Research Square concerning the legal status of any country, territory, city or area or of its authorities, or concerning the delimitation of its frontiers or boundaries. This map has been provided by the authors. 